\title{
A new approach to the study of quasi-normal modes of rotating stars
}

\author{
Valeria Ferrari ${ }^{1,2}$, Leonardo Gualtieri ${ }^{1,2}$, Stefania Marassi ${ }^{1,2}$ \\ 1 Dipartimento di Fisica "G. Marconi", Università "La Sapienza", I-00185 Roma, Italy \\ 2 INFN, Sezione di Roma, I-00185 Roma, Italy
}

\begin{abstract}
We propose a new method to study the quasi-normal modes of rotating relativistic stars. Oscillations are treated as perturbations in the frequency domain of the stationary, axisymmetric background describing a rotating star. The perturbed quantities are expanded in circular harmonics, and the resulting $2 \mathrm{D}$-equations they satisfy are integrated using spectral methods in the $(r, \theta)$-plane. The asymptotic conditions at infinity, needed to find the mode frequencies, are implemented by generalizing the standing wave boundary condition commonly used in the non rotating case. As a test, the method is applied to find the quasi-normal mode frequencies of a slowly rotating star.
\end{abstract}

PACS numbers: 04.40.Dg; 97.10.Sj

\section{INTRODUCTION}

Non radial oscillations of compact stars can be excited in several astrophysical events. For instance, after a neutron star (NS) is formed in a gravitational collapse, or in processes that may occur during its subsequent evolution; these include starquakes, glitches, interactions with a stellar companion, or phase transitions to quark matter or to a kaon and/or pion condensate, that may arise in the inner core of a NS if the density exceeds some critical value. All these phenomena induce perturbations which set the star in oscillation and, according to general relativity, gravitational waves (GWs) are one of the channels through which energy is dissipated.

In addition, due to rotation some modes may grow unstable through the Chandrasekhar-Friedman-Schutz mechanism (CFS instability) [1]; these instabilities may have important effects on the subsequent evolution of the star, and they may be associated to a further emission of GWs, the amount of which would depend on when and whether the growing modes are saturated by non-linear couplings or dissipative processes. It is therefore important to know at which frequencies a star pulsates emitting gravitational waves, and to study under which conditions the corresponding modes become unstable.

If one assumes that the star does not rotate, the mode frequencies can easily be computed by solving the equations of stellar perturbations, which have been formulated in the Sixties [2] and further developed in later years [3, 4]. These equations have been integrated for a large variety of equations of state proposed to describe matter in a NS [5]. These studies show that the identification of the frequency corresponding to the excitation of a stellar mode (for instance the fundamental mode which is likely to be the most energetic) in a detected gravitational signal, would allow to infer interesting information on the composition of the inner core of a NS and on the equation of state of matter at supranuclear densities.

However, all stars rotate, and our present knowledge of the quasi-normal mode (QNM) spectrum of rotating stars is far to be complete. The perturbative approach which works so fine in the non rotating case, when generalized to include rotation shows a high degree of complexity, even if the star is only slowly rotating [7, 8, 9, 10, 11, 12, 13, 14, 15, 16]. A major difficulty arises because, when using the standard spherical harmonics decomposition of the perturbed tensors, modes with different harmonic indexes couple, giving rise to an infinite set of dynamical, coupled equations. For this reason, in all studies based on this approach simplifying assumptions are introduced: either the couplings between oscillations with different values of the harmonic index $l$ are neglected, or Cowling's approximation is used (i.e. spacetime perturbations are neglected) $8,[9,10,11,12,13,14,[15]$. To our knowledge, the only place where the oscillations of a slowly rotating star are studied without making use of any of these restrictive assumptions is in [16] where, however, only $r$-modes have been considered.

An alternative approach consists in solving the equations describing a rotating and oscillating star in full general relativity, in time domain. However, current studies based on this approach also make use of strong simplifying assumptions, or restrict to particular cases. For instance, the Cowling approximation has been used in several papers [17]; in [18] only quasi-radial modes $(l=0)$ have been considered; in [19, 20] only the neutral mode (zero-frequency mode in the rotating frame) has been studied; in 21] only axisymmetric $(m=0)$ modes have been analysed, using the conformal flatness condition. In [22], the frequencies of axisymmetric modes $(m=0)$ with $l=0$ to $l=3$ have been computed for rapidly rotating relativistic polytropes, using the Cowling approximation; a comparison of the results for $l=0$ obtained in the Cowling approximation in [22], with those found in full GR in [18], shows that Cowling's approximation introduces large errors in the determination of the fundamental mode frequency.

In this article we develop a general method to find the quasi-normal mode frequencies of a rotating star. We perturb Einstein's equations about a stationary, axisymmetric background describing a rotating star. The perturbed 
quantities are expanded in circular harmonics $e^{\mathrm{i} m \phi}$. As we are looking for quasi-normal modes, we assume a time dependence $e^{-i \omega t}$, with $\omega$ complex. Due to the background symmetry, perturbations with different values of $\omega$ and $m$ are decoupled; thus, for assigned values of $(\omega, m)$, the perturbed equations to solve are a 2D-system of linear, differential equations in $r$ and $\theta$. In this paper we do not derive explicitly the perturbed equations in the general case of rapidly rotating stars, since they will be studied in a subsequent paper. Our goal here is to describe the general method.

Two are the main ingredients on which our method is based: i) the perturbed equations are integrated using spectral methods; ii) the boundary conditions at the center of the star and at radial infinity are implemented by suitably generalizing the standing wave approach which has been used to find the QNM frequencies of non-rotating stars [3, 4, 23, 24]. These points will be described in Section [I] To test our approach, in Section III we find the frequency of the fundamental mode of a slowly rotating, constant density star, as a function of the rotation rate. Concluding remarks are in Section IV.

\section{THE GENERAL METHOD}

The metric which we consider as a background is stationary and axially symmetric. It can be cast in the general form [25]

$$
\left(d s^{2}\right)^{(0)}=g_{\mu \nu}^{(0)} d x^{\mu} d x^{\nu}=e^{-\nu} d t^{2}+e^{\psi}(d \phi-\omega d t)^{2}+e^{\mu_{2}} d x_{2}^{2}+e^{\mu_{3}} d x_{3}^{2}
$$

where $\nu, \psi, \mu_{2}, \mu_{3}$ are functions of the coordinates $x_{2}, x_{3}$. In (1) there is still some gauge freedom, which allows to write the metric in a simpler form, like

$$
\left(d s^{2}\right)^{(0)}=e^{-\nu(r, \theta)} d t^{2}+e^{\psi(r, \theta)}(d \phi-w(r, \theta) d t)^{2}+e^{\mu(r, \theta)}\left(d r^{2}+r^{2} d \theta^{2}\right)
$$

as in [19], or like

$$
\left(d s^{2}\right)^{(0)}=e^{-\nu(r, \theta)} d t^{2}+e^{\lambda(r, \theta)} d r^{2}+e^{\mu(r, \theta)} r^{2}\left(d \theta^{2}+\sin ^{2} \theta(d \phi-w(r, \theta) d t)^{2}\right)
$$

as in [26], [27]. In the following we shall not specify explicitly the gauge, but we will require that some properties are satisfied; in particular we require: i) that the spacetime is described by the coordinates $(t, r, \theta, \phi)$, ii) that $\frac{\partial}{\partial t}, \frac{\partial}{\partial \phi}$ are Killing vectors associated with stationarity and axisymmetry respectively, and iii) that $\theta, \phi$ are polar coordinates on spheres, i.e. that the surfaces $t=$ const, $r=$ const are diffeomorphic (but not isomorphic) to 2-spheres. As a consequence of these assumptions (that are fulfilled by (2) and (3)) any tensor field defined on one of these surfaces can formally be expanded in tensor spherical harmonics, even if the spacetime is not spherically symmetric; this property will be useful in Section I A 2.

The metric and fluid velocity perturbations can be considered as tensor fields in this background; they are expanded in circular harmonics $e^{\mathrm{i} m \phi}$, and Fourier-transformed in time. Since perturbations belonging to different $m$ and different frequency $\omega$ do not couple, in what follows $m$ and $\omega$ will be considered as fixed, and perturbed quantities will be decomposed as follows

$$
\begin{aligned}
h_{\mu \nu}(t, r, \theta, \phi) & =h_{\mu \nu}^{m \omega}(r, \theta) e^{\mathrm{i} m \phi} e^{-\mathrm{i} \omega t} \\
\delta u_{\mu}(t, r, \theta, \phi) & =\delta u_{\mu}^{m \omega}(r, \theta) e^{\mathrm{i} m \phi} e^{-\mathrm{i} \omega t} \\
\delta \rho(t, r, \theta, \phi) & =\delta \rho^{m \omega}(r, \theta) e^{\mathrm{i} m \phi} e^{-\mathrm{i} \omega t} \\
\delta p(t, r, \theta, \phi) & =\delta p^{m \omega}(r, \theta) e^{\mathrm{i} m \phi} e^{-\mathrm{i} \omega t} .
\end{aligned}
$$

The frequency $\omega$ is, in general, complex.

By fixing the gauge, imposing $u^{\mu} u_{\mu}=-1$ and assigning an equation of state which relates $\delta p$ and $\delta \rho$, the sixteen quantities $h_{\mu \nu}^{m \omega}, \delta u_{\mu}^{m \omega}, \delta \rho^{m \omega}, \delta p^{m \omega}$ reduce to ten $\left\{H_{i}^{m \omega}(r, \theta)\right\}_{i=1, \ldots, 10}$. A possible gauge choice, which we call generalized Regge-Wheeler gauge, is described in Appendix A however, other gauges can be considered (see for instance [19]). For simplicity of notation, to hereafter we shall assume that the quantities $H_{i}^{m \omega}$ are scalars with respect to rotation (as they are, indeed, in the generalized Regge-Wheeler gauge, see Appendix A); however, every step of the approach we will describe can be applied also to tensorial quantities by a suitable generalization.

Einstein's equations, linearized about our stationary axisymmetric background, reduce to a system of partial differential equations (PDE) for the functions $H_{i}^{m \omega}$ in $r$ and $\theta$. To find the QNM frequencies, for assigned values of $m$ and $\omega$, we solve these equations by imposing that all metric functions are regular near the center of the star, that the Lagrangian perturbation of the pressure vanishes on the stellar surface, and that the solution at infinity behaves as a 
pure outgoing wave. The conditions at the center and at the surface of the star can be fulfilled for every value of $\omega$, but the outgoing wave condition at infinity is only consistent with a discrete set of (complex) frequencies $\left\{\omega_{i}\right\}$; such frequencies are the QNM.

We will now describe how to implement the boundary condition at infinity, and the numerical approach we use to solve the perturbed equations.

\section{A. The boundary condition at radial infinity}

In this section we shall generalize to the rotating case the standing wave approach [23, 24] used to find the QNM frequencies of non rotating stars. To this purpose, it is useful to remind how this method works.

\section{The standing wave approach for spherical stars}

First of all it is worth stressing that by this approach [23, 24] one can only determine the QNM frequencies of slowly damped modes, like the $f$-, $p$-, and $r$-modes; it cannot be applied to highly damped modes, like stellar $w$-modes or black hole's QNM.

It is known that the equations describing the perturbations of a non rotating, spherical star can be separated by expanding the perturbed tensors in tensorial spherical harmonics; outside the star these equations reduce to those describing Schwarzschild perturbations, and they can be reduced to the Regge-Wheeler [28] and the Zerilli [29] equations, for two suitably defined functions which we both indicate as $Z^{l m}(r, \omega)$. The two wave equations have the following form

$$
\frac{d^{2} Z^{l m}(r, \omega)}{d r_{*}^{2}}+\left[\omega^{2}-V(r)\right] Z^{l m}(r, \omega)=0, \quad l \geq 2,
$$

where $r_{*}$ is the usual tortoise coordinate and $V(r)$ is a short range potential. The QNM frequencies are the values of the complex frequency $\omega$ for which the solutions of Equation (5), found by imposing appropriate boundary conditions at the surface of the star, behave as pure outgoing waves at radial infinity, i.e.

$$
Z^{l m}(r, \omega)=A_{\text {out }}^{l m}(\omega) e^{\mathrm{i} \omega r_{*}} \quad \text { as } \quad r_{*} \longrightarrow \infty .
$$

The standing wave approach consists in the following. Let us assume that $Z^{l m}(r, \omega)$ is an analytic function of the complex variable $\omega=\sigma-\mathrm{i} / \tau$, and be $\omega_{0}=\sigma_{0}-\mathrm{i} / \tau_{0}$ the frequency of a QNM, with $\left|1 / \tau_{0}\right| \ll \sigma_{0}$. In general, at radial infinity the solution of Equation (5) is a superposition of ingoing and outgoing waves, i.e.

$$
Z^{l m}(r, \omega)=A_{i n}^{l m}(\omega) e^{-\mathrm{i} \omega r_{*}}+A_{\text {out }}^{l m}(\omega) e^{\mathrm{i} \omega r_{*}} .
$$

If $\omega=\omega_{0}$, by definition $A_{i n}^{l m}(\omega)=0$; since $Z^{l m}$ is analytic and since $\left|1 / \tau_{0}\right| \ll \sigma_{0}$, we can expand $A_{i n}^{l m}(\sigma)$ near the real $\sigma_{0}$ as follows

$$
A_{i n}^{l m}\left(\omega_{0}\right)=A_{i n}^{l m}\left(\sigma_{0}\right)-\frac{\mathrm{i}}{\tau_{0}} A_{i n}^{l m}{ }^{\prime}\left(\sigma_{0}\right),
$$

where " '" indicates differentiation with respect to $\sigma$; then, by imposing $A_{i n}^{l m}\left(\omega_{0}\right)=0$ we find

$$
A_{i n}^{l m}{ }^{\prime}\left(\sigma_{0}\right)=-\mathrm{i} \tau_{0} A_{i n}^{l m}\left(\sigma_{0}\right) \text {. }
$$

Using this relation the function $A_{i n}^{l m}(\sigma)$, near $\sigma=\sigma_{0}$ (with $\sigma$ and $\sigma_{0}$ real), can be written as

$$
A_{i n}^{l m}(\sigma)=A_{i n}^{l m}\left(\sigma_{0}\right)+\left(\sigma-\sigma_{0}\right) A_{i n}^{l m}{ }^{\prime}\left(\sigma_{0}\right)=-\mathrm{i} \tau_{0} A_{i n}^{l m}\left(\sigma_{0}\right)\left[\left(\sigma-\sigma_{0}\right)+\frac{\mathrm{i}}{\tau_{0}}\right] \text {, }
$$

from which it follows

$$
\left|A_{i n}^{l m}(\sigma)\right|^{2}=B^{2}\left[\left(\sigma-\sigma_{0}\right)^{2}+\frac{1}{\tau_{0}^{2}}\right],
$$

where $B$ is a constant which does not depend on $\sigma$. Thus, to find the frequencies of the QNM it is sufficient to integrate the wave equation (5) for real values of the frequency $\sigma$, and find the values $\sigma_{i}$ for which the amplitude of the standing wave (11) has a minimum: these are the QNM frequencies. The corresponding damping times $\tau_{i}$ can be found through a quadratic fit of $\left|A_{i n}^{l m}(\sigma)\right|^{2}$. 


\section{The standing wave approach for rotating stars}

Let us now consider a rotating star. As discussed above, with a suitable choice of the gauge the relevant perturbed quantities reduce to a set of quantities which behave as scalars with respect to rotation: $\left\{H_{i}^{m \omega}(r, \theta)\right\}_{i=1, \ldots, 10}(\omega$ complex). They must satisfy a set of PDE, obtained by linearizing Einstein's equation, which can be integrated once the values of these quantities are assigned at the center of the star, i.e. on a sphere of radius $r_{0} \ll R$ (hereafter $R$ is the stellar radius)

$$
H_{i}^{m \omega}\left(r_{0}, \theta\right)=H_{0 i}^{m \omega}(\theta) .
$$

The $H_{0 i}^{m \omega}(\theta)$ are subject to constraints, which arise from the analytical expansion in powers of $r$ of the perturbed equations, from the assumption of regularity of the spacetime as $r \rightarrow 0$, and from the requirement that the Lagrangian pressure perturbation must vanish on the surface of the star. These constraints reduce the number of independent quantities from the ten $H_{0 i}^{m} \omega(\theta)$ to a smaller number, say $N$, i.e. $\left\{\hat{H}_{0 j}^{m}(\theta)\right\}_{j=1, \ldots, N}$. Being these quantities defined on a sphere $r=r_{0}$, they can formally be decomposed in spherical harmonics

$$
\hat{H}_{0 j}^{m}(\theta)=\sum_{l=|m|}^{L} \hat{H}_{j}^{l m} Y^{l m}(\theta, 0),
$$

where the expansion is truncated at $l=L$. Therefore the independent solutions of the perturbed equations correspond to the following set of $N \cdot[L-|m|+1]$ constants

$$
\left\{\hat{H}_{j}^{l m}\right\} \quad \text { with } \quad j=1, \ldots, N \quad \text { and } \quad l=|m|, \ldots, L .
$$

Given these constants, the perturbed equations for the functions $H_{i}^{m \omega}(r, \theta)$ can be integrated for $r \geq r_{0}$.

In the wave zone, far away from the star, the far field limit expansion of the metric describing a rotating star shows that the metric reduces to the Schwarzschild solution (see for instance [30], Chap. 19). This occurs because terms due to rotation decrease faster than the "Schwarzschild-like" components. Therefore, as when dealing with Schwarzschild perturbations, in this asymptotic region we can define the gauge invariant Zerilli and Regge-Wheeler functions, $Z_{Z e r}^{l m}(r, \omega)$ and $Z_{R W}^{l m}(r, \omega)$, in terms of the perturbed metric tensor, expanded in tensorial spherical harmonics with $l \geq 2$. This tensor is found by integrating the equations describing the perturbed spacetime outside the rotating star. The well known asymptotic behaviour of $Z_{Z e r}^{l m}(r, \omega)$ and $Z_{R W}^{l m}(r, \omega)$ is

$$
\begin{aligned}
& Z_{Z e r}^{l m}(r, \omega)=A_{Z e r i n}^{l m}(\omega) e^{-\mathrm{i} \omega r_{*}}+A_{Z \text { er out }}^{l m}(\omega) e^{\mathrm{i} \omega r_{*}} \\
& Z_{R W}^{l m}(r, \omega)=A_{R W \text { in }}^{l m}(\omega) e^{-\mathrm{i} \omega r_{*}}+A_{R W \text { out }}^{l m}(\omega) e^{\mathrm{i} \omega r_{*}} .
\end{aligned}
$$

A (complex) frequency $\omega_{0}$ belongs to a quasi-normal mode if, for an assigned value of $m$, the following condition is satisfied for any $l$ :

$$
A_{Z \operatorname{lin} \text { in }}^{l m}\left(\omega_{0}\right)=A_{R W \text { in }}^{l m}\left(\omega_{0}\right)=0 \quad \forall l
$$

i.e. if the set of $2 \cdot[L-|m|+1]$ constants

$$
\left\{A_{Z \text { er in }}^{l m}(\omega), A_{R W \text { in }}^{l m}(\omega)\right\} \quad \text { with } \quad l=|m|, \ldots, L
$$

vanishes.

It should be stressed that this is a big difference with respect to the non rotating case: in that case each mode belongs to a single, assigned value of $l$, and there is degeneracy in $m$.

For each assigned value of $m$, we define the vectors

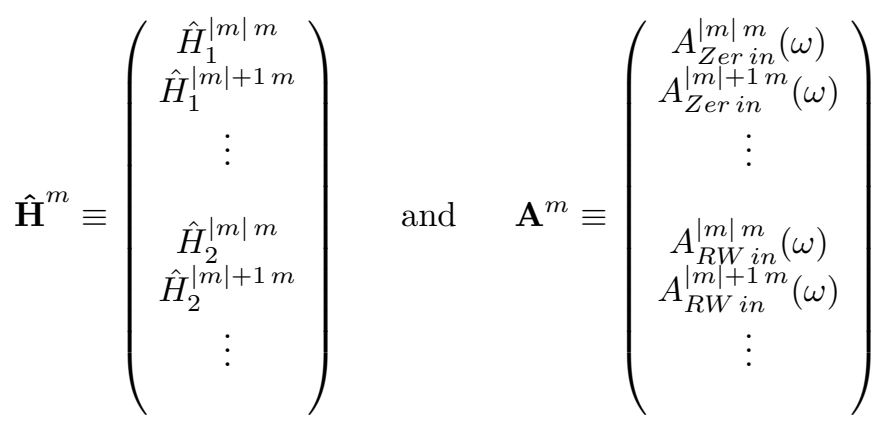


the dimensionality of which is $N \cdot[L-|m|+1]$ and $2 \cdot[L-|m|+1]$, respectively. Since the perturbed equations are linear, these vectors are related by the matrix equation

$$
\mathbf{A}^{m}(\omega)=\mathbf{M}^{m}(\omega) \hat{\mathbf{H}}^{m}
$$

the constants $\hat{H}_{j}^{l m}$ do not depend on $\omega$. The coefficients of the complex matrix $\mathbf{M}^{m}(\omega)$ have to be evaluated by integrating the perturbed equations.

Equation (16), which identifies the QNM eigenfrequencies, can be written as

$$
\mathbf{M}^{m}\left(\omega_{0}\right) \hat{\mathbf{H}}^{m}=0 \quad \forall \hat{\mathbf{H}}^{m} .
$$

A discrete set of QNM's exists if the matrix $\mathbf{M}$ is square, i.e. if $N=2$. Thus, equation (20) is equivalent to impose

$$
\operatorname{det}\left(\mathbf{M}^{m}\left(\omega_{0}\right)\right)=0 .
$$

As we will show in the Appendices, by counting the number of independent equations in the cases of spherical stars and of slowly rotating stars we find indeed $N=2$. We expect the same to hold also for rapidly rotating stars.

Let us now restrict the frequency to the real axis. Furthermore, we normalize the constants $\hat{\mathbf{H}}^{m}$ so that the solutions $H_{i}^{m \sigma}(r, \theta)$ (and consequently $\left.Z_{Z e r}^{l m}(r, \sigma), Z_{R W}^{l m}(r, \sigma)\right)$ are real; this is always possible, because the perturbed Einstein equations in the frequency domain have real coefficients, as long as $\sigma$ is real (if we assume that the fluid is non dissipative, so that the equations in time domain are time-symmetric). Thus, in the wave zone we have

$$
\begin{aligned}
& Z_{Z e r}^{l m}(r, \sigma)=A_{Z \text { er in }}^{l m}(\sigma) e^{-\mathrm{i} \sigma r_{*}}+A_{Z \text { er out }}^{l m}(\sigma) e^{\mathrm{i} \sigma r_{*}} \in \mathbb{R} \\
& Z_{R W}^{l m}(r, \sigma)=A_{R W \text { in }}^{l m}(\sigma) e^{-\mathrm{i} \sigma r_{*}}+A_{R W \text { out }}^{l m}(\sigma) e^{\mathrm{i} \sigma r_{*}} \in \mathbb{R} .
\end{aligned}
$$

The ingoing wave amplitudes, $A_{Z \text { er in }}^{l m}$ and $A_{R W \text { in }}^{l m}$, can be found, as shown in [24], by evaluating $Z_{Z e r}$ and $Z_{R W}$ at different values of $r_{*}$, fitting the two functions as a superposition of $\sin \sigma r_{*}$ and $\cos \sigma r_{*}$. It should be noted that although $\hat{\mathbf{H}}$ are real, the quantities $A_{Z \text { er in }}^{l m}, A_{R W \text { in }}^{l m}$ are complex (they satisfy the conditions $A_{Z \text { er out }}^{l m}=\left(A_{Z \text { er in }}^{l m}\right)^{*}$, $\left.A_{R W \text { out }}^{l m}=\left(A_{R W \text { in }}^{l m}\right)^{*}\right)$, thus $\mathbf{M}$ is complex. For $\sigma$ real, the vectors $\hat{\mathbf{H}}^{m}, \mathbf{A}^{m}$ are related by

$$
\mathbf{A}^{m}(\sigma)=\mathbf{M}^{m}(\sigma) \hat{\mathbf{H}}^{m} .
$$

By expanding equation (21) about $\omega_{0}=\sigma_{0}-\mathrm{i} / \tau_{0}$ (with $\left|1 / \tau_{0}\right| \ll \sigma_{0}$ ) as we did for spherical stars, we find that if $\sigma \sim \sigma_{0}$

$$
\operatorname{det} \mathbf{M}^{m}(\sigma)=\operatorname{det} \mathbf{M}\left(\sigma_{0}\right)\left[1-\mathrm{i} \tau_{0}\left(\sigma-\sigma_{0}\right)\right] \quad \Rightarrow \quad\left|\operatorname{det} \mathbf{M}^{m}(\sigma)\right| \propto \sqrt{\left(\sigma-\sigma_{0}\right)^{2}+{\frac{1}{\tau_{0}}}^{2}} .
$$

Thus, the QNM frequencies are found by evaluating the (complex) matrix $\mathbf{M}$ for real values of the frequency $\sigma$, finding the minima of the modulus of its determinant. The standing wave approach has thus been generalized to rotating stars.

\section{B. Spectral methods for stellar oscillations}

We now summarize the procedure to solve the 2D-perturbed equations using spectral methods. They are, indeed, very powerful to solve 2D-differential equations, and particularly useful to implement boundary conditions. For a general discussion on spectral methods we refer the reader to [31].

\section{Chebyshev polynomials}

We expand all functions of $r$ in Chebyshev polynomials:

$$
f(x)=\sum_{n=0}^{\infty} a_{n} T_{n}(x) \quad \text { with } \quad T_{n}(\cos (u))=\cos (n u) \quad n=0,1, \ldots
$$

which satisfy the orthogonality relations

$$
\int_{-1}^{1} T_{m}(x) T_{n}(x) \frac{d x}{\sqrt{1-x^{2}}}=\frac{\pi}{2}\left(1+\delta_{m 0}\right) \delta_{m n}
$$


$(n, m=0,1, \ldots, i=1,2, \ldots)$. The variable $x \in[-1,1]$ is related to $r \in[a, b]$ by the following equation

$$
x=\frac{2 r-b-a}{b-a} \in[-1,1] .
$$

- Integrals on Chebyshev polynomials can be evaluated using the Gaussian quadrature method [32]: truncating the polynomial expansion at $n=K$ we get

$$
\int_{-1}^{1} g(x) \frac{d x}{\sqrt{1-x^{2}}}=\frac{\pi}{K+1} \sum_{n=0}^{K} g\left(x_{n}\right),
$$

where the collocation points are

$$
x_{n}=\cos \left(\frac{\pi(n+1 / 2)}{K+1}\right) \quad n=0,1, \ldots, K .
$$

- The derivative of a function can be expressed as follows

$$
f^{\prime}(x)=\sum_{n, m}^{0, K}\left(D_{m n} a_{n}\right) T_{m}
$$

with

$$
\begin{aligned}
D_{K n} & =0 \\
D_{k-1 n} & =D_{k+1 n}+2 k \delta_{k n} \quad k=2, \ldots, K \\
D_{0 n} & =\frac{1}{2}\left[D_{2 n}+2 \delta_{1 n}\right] .
\end{aligned}
$$

- Given a function $V(x)$, and a function $f(x)$ with Chebyshev expansion

$$
f(x)=\sum_{n=0}^{K} a_{n} T_{n}(x)
$$

the expansion of $V(x) f(x)$ is

$$
V(x) f(x)=\sum_{n=0}^{K} b_{n} T_{n}(x),
$$

where $b_{n}=V_{n m} a_{m}$ with

$$
V_{n m}=\frac{2-\delta_{m 0}}{K+1} \sum_{k=0}^{K} V\left(x_{k}\right) T_{n}\left(x_{k}\right) T_{m}\left(x_{k}\right) .
$$

\section{Associated Legendre polynomials}

We expand all functions of $\theta$ in the basis of the associated Legendre polynomials

$$
P^{l m}(y)=(-1)^{m}\left(1-y^{2}\right)^{m / 2} \frac{d^{m}}{d y^{m}} P^{l}(y),
$$

with

$$
y=\cos \theta \in[-1,1]
$$

and $m$ fixed. They are related to scalar spherical harmonics:

$$
\begin{array}{ll}
Y^{l m}(\theta, \phi)=\sqrt{\frac{2 l+1}{4 \pi} \frac{(l-m) !}{(l+m) !}} P^{l m}(\cos \theta) e^{\mathrm{i} m \phi} & \text { if } m \geq 0 \\
Y^{l m}(\theta, \phi)=(-1)^{l}\left(Y^{l-m}\right)^{*} & \text { if } m<0 .
\end{array}
$$


Therefore, expanding a function in circular harmonics $e^{\mathrm{i} m \phi}$ and in associated Legendre polynomials is equivalent to expand it in spherical harmonics. $P^{l m}$ 's are eigenfunctions of the Laplacian operator,

$$
\left(\partial_{\theta}^{2}+\cot \theta \partial_{\theta}-\frac{m^{2}}{\sin ^{2} \theta}\right) P^{l m}=-l(l+1) P^{l m},
$$

and, assuming for simplicity of notation $m \geq 0$, have the following asymptotic behaviour near the $z$-axis (see (35) ):

$$
P^{l m} \sim\left(1-y^{2}\right)^{m / 2}=(\sin \theta)^{m} \quad \text { if } \quad \theta \simeq 0, \pi .
$$

This is the asymptotic behaviour of any function of $\theta$ which is regular on the $z$-axis. Let us consider a function $f(r, \theta, \phi)$, regular in $r=0$ and on the $z$-axis; let us expand it in circular harmonics

$$
f(r, \theta, \phi)=\sum_{m} f^{m}(r, \cos \theta) e^{\mathrm{i} m \phi} .
$$

The regularity of $f(r, \theta, \phi)$ near the $z$-axis implies that (see for instance [33])

$$
\lim _{\theta=0, \pi} \frac{f^{m}(r, \cos \theta)}{(\sin \theta)^{m}}=\text { finite. }
$$

Therefore, the $f^{m}(r, \cos \theta)$ can be expanded in the polynomials $\left\{P^{l m}\right\}_{l=|m|, \ldots}$ with $m$ fixed:

$$
f^{m}(r, y)=\sum_{l=|m|}^{\infty} a^{l m}(r) P^{l m}(y)
$$

Thus, associated Legendre polynomials $P^{l m}$ (with $m$ fixed) are a complete basis for all functions of $\theta$ with the asymptotic behaviour (39).

In order to apply the Gaussian quadrature method to associated Legendre's polynomials, we notice that the polynomials

$$
\bar{P}^{l m}(y) \equiv \frac{P^{l m}(y)}{\left(1-y^{2}\right)^{m / 2}},
$$

form, for each $m$, a complete basis, with orthogonality relation

$$
\int_{-1}^{1} \bar{P}^{l m}(y) \bar{P}^{l^{\prime} m}(y)\left(1-y^{2}\right)^{m} d y=\frac{2}{2 l+1} \frac{(l+m) !}{(l-m) !} \delta_{l l^{\prime}} .
$$

The $\bar{P}^{l m}(y)$ 's are a particular case of Jacobi's polynomials $J_{l}^{(\alpha, \beta)}(y)$ defined by [32]

$$
\int_{-1}^{1} J_{l}(y) J_{l^{\prime}}(y)(1-y)^{\alpha}(1+y)^{\beta} \propto \delta_{l l^{\prime}}
$$

where $\alpha=\beta=m$. Therefore, Gaussian integration takes the form

$$
\int_{-1}^{1} f^{m}(y) P^{l m}(y) d y=\sum_{k} w_{k} \frac{f\left(y_{k}\right) P^{l m}\left(y_{k}\right)}{1-y_{k}^{2}}
$$

where $y_{k}$ and $w_{k}$ are the collocation points and weights for the Jacobi polynomials with $\alpha=\beta=m$. In particular, the coefficients of the expansion (42) are

$$
a^{l m}=\frac{2 l+1}{2} \frac{(l-m) !}{(l+m) !} \sum_{k} w_{k} \frac{f\left(y_{k}\right) P^{l m}\left(y_{k}\right)}{1-y_{k}^{2}} .
$$




\section{Differential equations and boundary conditions}

Let us consider a one-dimensional, first order, differential equation

$$
Z^{\prime}(x)+V(x) Z(x)=0
$$

with $x \in[-1,1]$. If we expand $Z(x)$ in the basis of Chebyshev polynomials, $T_{n}(x)$, truncating the expansion at $n=K$, i.e.

$$
Z(x)=\sum_{n=0}^{K} a_{n} T_{n}(x)
$$

the differential equation (48) becomes an algebraic equation:

$$
\sum_{k=0}^{K}\left(D_{n k}+V_{n k}\right) a_{k}=0 \quad(n=0, \ldots, K),
$$

where $D_{n k}$ and $V_{n k}$ are defined in (31), (34).

The boundary conditions needed to solve Equation (48) can be implemented using the so-called $\tau$-method: we cut the last row of (50), i.e. we set

$$
\sum_{k=0}^{K}\left(D_{n k}+V_{n k}\right) a_{k}=0 \quad(n=0, \ldots, K-1),
$$

and replace the row with the boundary condition; for instance, if we know that $Z\left(x_{0}\right)=z_{0}$, the last row of the matrix equation will be replaced with

$$
\sum_{k=0}^{K} T_{k}\left(x_{0}\right) a_{k}=z_{0}
$$

The differential equation (48), with the boundary condition $Z\left(x_{0}\right)=z_{0}$, thus reduces to a matrix equation which can be solved by $L U$ decomposition [32].

This approach can easily be generalized to higher order differential equations (by replacing more rows for the associated boundary conditions), to systems of coupled differential equations, and to partial differential equations in $r, \theta$. In this case, each function is expanded in the basis $\left\{T_{n}(x), P^{l m}(y)\right\}_{n, l}$ as follows

$$
f^{m}(x, y)=\sum_{n=0}^{K} \sum_{l=|m|}^{L} a_{n}^{l m} T_{n}(x) P^{l m}(y)
$$

where sums over Chebyshev's and associated Legendre's polynomials are truncated to the orders $K$ and $L$, respectively; the coefficients $a_{n}^{l m}$ can be organized as the components of a vector, defining the collective index

$$
i(l, n)=[l-|m|](K+1)+n+1=1,2, \ldots,[L-|m|+1](K+1)
$$

and setting

$$
\hat{a}_{i}^{m}=\hat{a}_{i(l, n)}^{m} \equiv a_{n}^{l m}
$$

In terms of the expansion (53), PDEs in $r, \theta$ (in our case, the equations which describe the perturbations of rotating stars) transform into an algebraic equation. As an example of the double expansion (53), in Appendix A3 we show how to solve the Regge-Wheeler equation as a partial differential equation in $r, \theta$.

\section{A TEST OF THE METHOD: OSCILLATIONS OF SLOWLY ROTATING STARS}

As a test of our method, we have solved the equations which describe the perturbations of a slowly rotating stars. Following [27], in this case the metric and fluid velocity of the unperturbed star are

$$
\begin{aligned}
\left(d s^{2}\right)^{(0)} & =g_{\mu \nu}^{(0)} d x^{\mu} d x^{\nu}=e^{-\nu(r)} d t^{2}+e^{\lambda(r)} d r^{2}+r^{2}\left(d \theta^{2}+\sin ^{2} \theta d \phi^{2}\right)-2 \omega(r) r^{2} \sin ^{2} \theta d t d \phi \\
u^{(0) \mu} & =\left(e^{-\nu / 2}, 0,0, \Omega e^{-\nu / 2}\right)
\end{aligned}
$$


where $\omega(r)$ describes the dragging of the inertial frames, and all quantities are expanded at first order in the angular velocity of the star, $\Omega$. We introduce a rotation parameter $\epsilon$ defined by

$$
\epsilon=\Omega / \sqrt{M / R^{3}} .
$$

In the metric (56) spherical symmetry is broken only by the term $\omega$; when Einstein's equations are perturbed about (56), only terms which are linear in $\omega$ are retained, i.e. we keep terms up to order $O(\epsilon)$. As a consequence, perturbations with index $l$ are coupled with perturbations with indexes $l \pm 1$ through terms that are of order $O(\epsilon)$, the analytical form of which can explicitly be derived. Therefore, when we transform the perturbed equations using the double spectral decomposition described in Section IIB, the resulting algebraic equations have a particularly simple form: the relevant matrix is "almost-block-diagonal", each block corresponding to one value of $l$; the off-diagonal blocks couple $l \leftrightarrow l \pm 1$.

These equations can also be obtained in a different way, i.e. by expanding in Chebyshev polynomials the system of ordinary differential equations in $r$ derived by Kojima in 7] (hereafter, K1). This follows from the fact that Kojima's equations are derived by expanding the perturbed Einstein's equations in spherical harmonics.

The general structure of Kojima's equations is the following:

$$
\begin{aligned}
\mathcal{L}^{p o l}\left[H_{0}^{l m}, K^{l m} ; \sigma\right] & =m \mathcal{E}\left[H_{0}^{l m}, K^{l m} ; \sigma\right]+\mathcal{F}^{( \pm)}\left[Z_{R W}^{l \pm 1 m} ; \sigma\right] \\
\mathcal{L}^{a x}\left[Z_{R W}^{l m} ; \sigma\right] & =m \mathcal{N}\left[Z_{R W}^{l m} ; \sigma\right]+\mathcal{D}^{( \pm)}\left[H_{0}^{l \pm 1 m}, K^{l \pm 1 m} ; \sigma\right] .
\end{aligned}
$$

Here $\mathcal{L}^{\text {pol }}, \mathcal{L}^{a x}$ are operators of order $O\left(\epsilon^{0}\right)$, which describe the perturbations of the star in the non rotating case; $\mathcal{E}, \mathcal{F}^{( \pm)}, \mathcal{N}, \mathcal{D}^{( \pm)}$are $O\left(\epsilon^{1}\right)$ operators, which provide the corrections due to rotation. These equations have been integrated numerically in [8] (see also [9]) using a very strong simplification: the couplings $l \leftrightarrow l \pm 1$ were neglected (i.e. $\mathcal{F}^{( \pm)}$and $\mathcal{D}^{( \pm)}$were set to zero).

Moreover, the equations were solved iteratively, finding the solution for $\epsilon=0$ first, and then replacing it in the terms $\mathcal{E}\left[H_{0}^{l m}, K^{l m} ; \sigma\right]$ and $\mathcal{N}\left[Z_{R W}^{l m} ; \sigma\right]$ of eqs. (58). In this way, the right-hand sides of eqs. (58) become source terms.

We stress that with our approach we do not need these simplifications anymore; in particular, we do not need to neglect the couplings, because we can handle the mixing among perturbations with different $l$ 's using the spectral methods and the generalized standing wave approach.

It should also be mentioned that, when coupling terms are included in the perturbed equations, in order to have a good numerical behaviour of the perturbations near the center of the star we need to use a set of variables (in particular the $O\left(\epsilon^{1}\right)$ terms) different from that used in [9]. The equations for the new variables are given explicitly in Appendix B

\section{A. Comparison with existing results}

As mentioned above, in [8] (hereafter K2) the equations of stellar perturbations have been integrated for a slowly rotating star, neglecting $l \leftrightarrow l \pm 1$ couplings, and the QNM frequencies have been found; to reproduce these results we have used the same set of variables as in K1, the same equation of state (EOS) i.e. the polytropic $\operatorname{EOS~} p=K \rho^{2}$, and we have computed the fundamental mode ( $f$-mode) frequency, $\sigma_{f}$.

In K2 the real and imaginary parts of $\sigma_{f}$ are fitted as functions of the rotation parameter $\epsilon$ as follows:

$$
\begin{aligned}
\sigma_{f}^{R} & =\sigma_{0}^{R}\left(1+m \epsilon \sigma_{R}^{\prime}\right)+O\left(\epsilon^{2}\right), \\
\sigma_{f}^{I} & =\sigma_{0}^{I}\left(1+m \epsilon \sigma_{I}^{\prime}\right)+O\left(\epsilon^{2}\right) .
\end{aligned}
$$

The value of $\sigma_{0}^{R}$ we find, properly normalized, is plotted versus the stellar compactness, $M / R$, in Figure 1 a). The values are in excellent agreement with the results shown in Figure 1 of K2, for $n=1$.

The correction due to rotation, $\sigma_{R}^{\prime}$, is plotted versus $M / R$ in Figure $1 \mathrm{~b}$ ) for different values of $\epsilon$. We find that for $\epsilon \lesssim 10^{-3}$ the corresponding curves are indistinguishable, and coincide with the $n=1$ curve shown in Figure 1 of K2. However, for $\epsilon \gtrsim 10^{-3}$ different $\epsilon$ correspond to different curves, and the fit (59) becomes inaccurate: $\sigma_{R}^{\prime}$ is no longer a constant, and further corrections to (59) are of order $O\left(\epsilon^{2}\right)$, as expected theoretically.

In Figure 2 we plot the imaginary part of the $f$-mode frequency, $\sigma_{0}^{I}$, and the corresponding rotational correction, $\sigma_{I}^{\prime}$, as in Figure 1. $\sigma_{I}^{\prime}$ is plotted only for $\epsilon \geq 10^{-2}$, because for smaller values our numerical approach becomes inaccurate. They agree with the values given in Figure 2 of K2, with differences of order $O\left(\epsilon^{2}\right)$. 

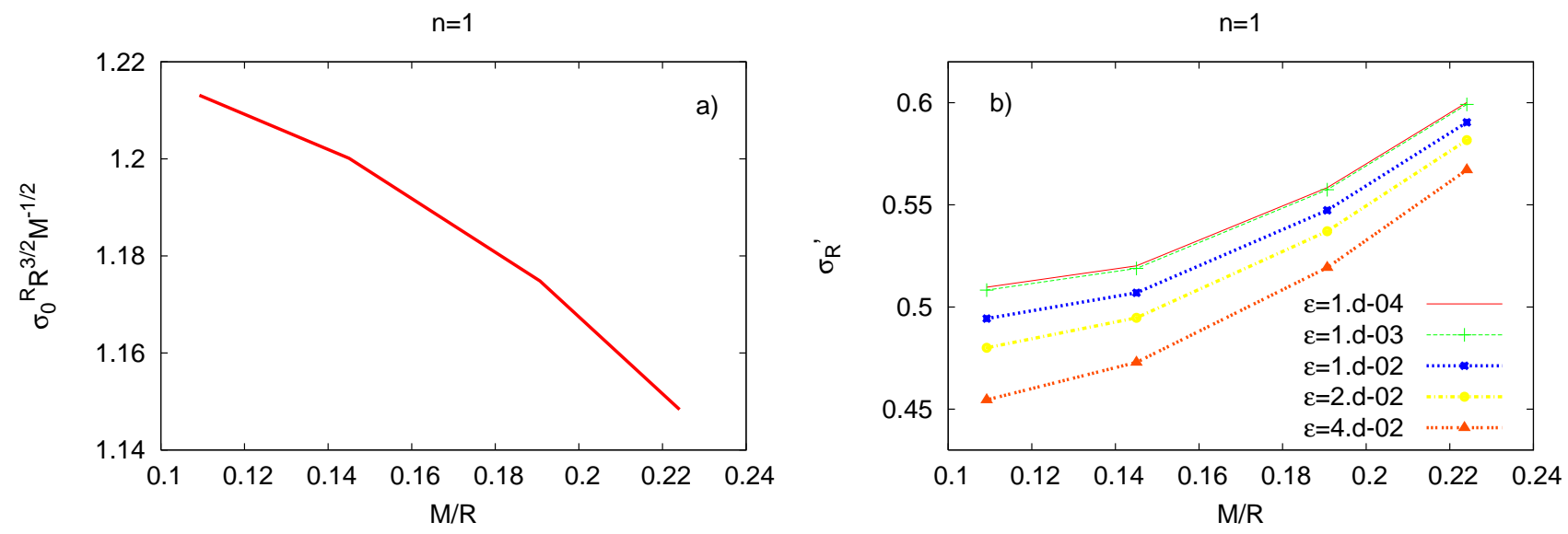

FIG. 1: The real part of the $f$-mode frequency of a non rotating, polytropic star with $n=1$, is plotted as a function of the stellar compactness $M / R$ (a); the frequency shift due to rotation, $\sigma_{R}^{\prime}$ is plotted versus compactness for different values of $\epsilon$ (b). As in K2, couplings among different $l$ 's are neglected.
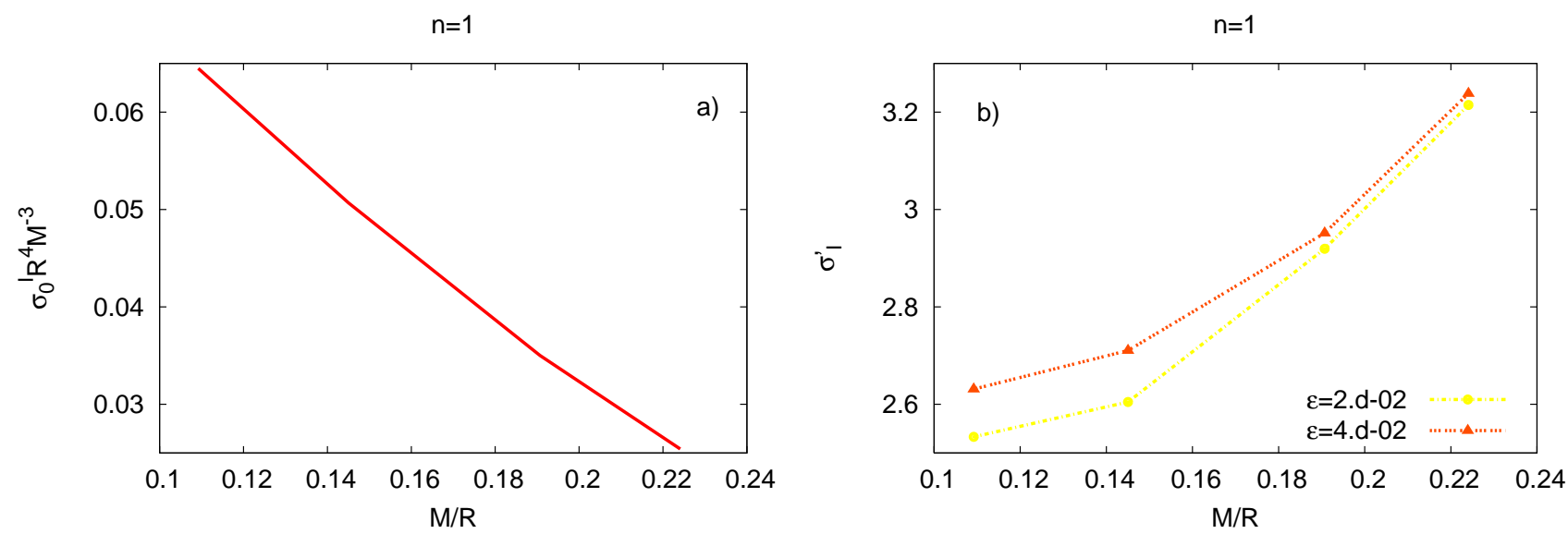

FIG. 2: The imaginary part of the $f$-mode frequency (a) and the corrections due to rotation (b) are plotted as in Figure 1

\section{B. Including the couplings}

We now apply our method to solve eqs. (58) in full, i.e. including couplings among different l's. The numerical implementation of the equations presents a problem: there are terms in the equations which depend on $\left(\frac{\partial p}{\partial \rho}\right)^{2}$ and on $\left(\frac{\partial^{2} p}{\partial \rho^{2}}\right)$, which strongly diverge on the stellar surface. This divergence is particularly problematic when spectral methods are used, but it can be cured through a regularization procedure [33]. Such regularization goes beyond the scope of the present paper, where we only want to discuss a simple implementation of our approach. Therefore, we solve the perturbed equations in the case of a constant density, slowly rotating star, such that the divergent terms vanish.

We consider two background models: $A$, with $M / R=0.2$ and $B$, with $M / R=0.1$. Mass and radius forałssigned values of the central density are given in Table [. The explicit form of the equations and the boundary conditions in

\begin{tabular}{|c|c|c|c|c|}
\hline & $\rho\left(\mathrm{g} / \mathrm{cm}^{3}\right)$ & $M / M_{\odot}$ & $R(\mathrm{Km})$ & $M / R$ \\
\hline$A$ & $10^{15}$ & 1.11 & 8.08 & 0.2 \\
\hline$B$ & $10^{15}$ & 0.40 & 5.75 & 0.1 \\
\hline
\end{tabular}

TABLE I: Parameters of the constant density stellar models $A$ and $B$ we use as a background. 
$r=0$ and $r=R$ are discussed in Appendix B. As mentioned above, when couplings are included the equations derived in $\mathrm{K} 1$ are very unstable when integrated near the center. For this reason we introduce a new set of variables, which satisfy a new set of equations shown in Appendix $\mathrm{B}$, the appropriate boundary conditions in $r=0$ and $r=R$ are also shown. Once we assign the value of the harmonic index $m$, these equations couple polar and axial perturbations with $|m| \leq l \leq L$.

The equations have been integrated for $m= \pm 2$. We do not set $|m|<2$ because in that case dipolar $(l=1)$ perturbations have to be taken into account, which are described by equations different from those we consider in this paper. We would like to stress that rotational corrections to mode frequencies with $m \neq 0$ are much larger than those to mode frequencies with $m=0$. In K2, Kojima suggested that, at lowest order in $\epsilon$, the frequency shift is proportional to $m$. This is consistent with the results of our numerical integration: the relative frequency shifts found in [21], where $m=0$ perturbations were studied in full general relativity, are an order of magnitude smaller than the relative shifts we find for $m= \pm 2$.

If the star does not rotate, for any assigned value of $l$ there is a corresponding $f$-mode frequency, which is the same for all $m$ 's. If the star rotates, due to the couplings the $f$-mode belonging to an assigned $l$ acquires contributions from different $l$ 's, and its frequency and damping time change. Furthermore, the degeneracy in $m$ is broken by rotation.

The real part of the $f$-mode frequency, $\nu_{f}=\sigma_{f}^{R} /(2 \pi)$, is plotted as a function of the rotation parameter in Figure 3 for the two considered stellar models. The solid line represents the frequency of the $l=2 f$-mode of the non-rotating star. The dashed lines are the frequencies of the lowest lying fundamental mode of the rotating star, with $m=2$ and $m=-2$, assuming $L=4$. Our calculations refer to $\epsilon \leq 0.05$, since for higher values the slow rotation approximation becomes inaccurate and the results cannot be trusted anymore.
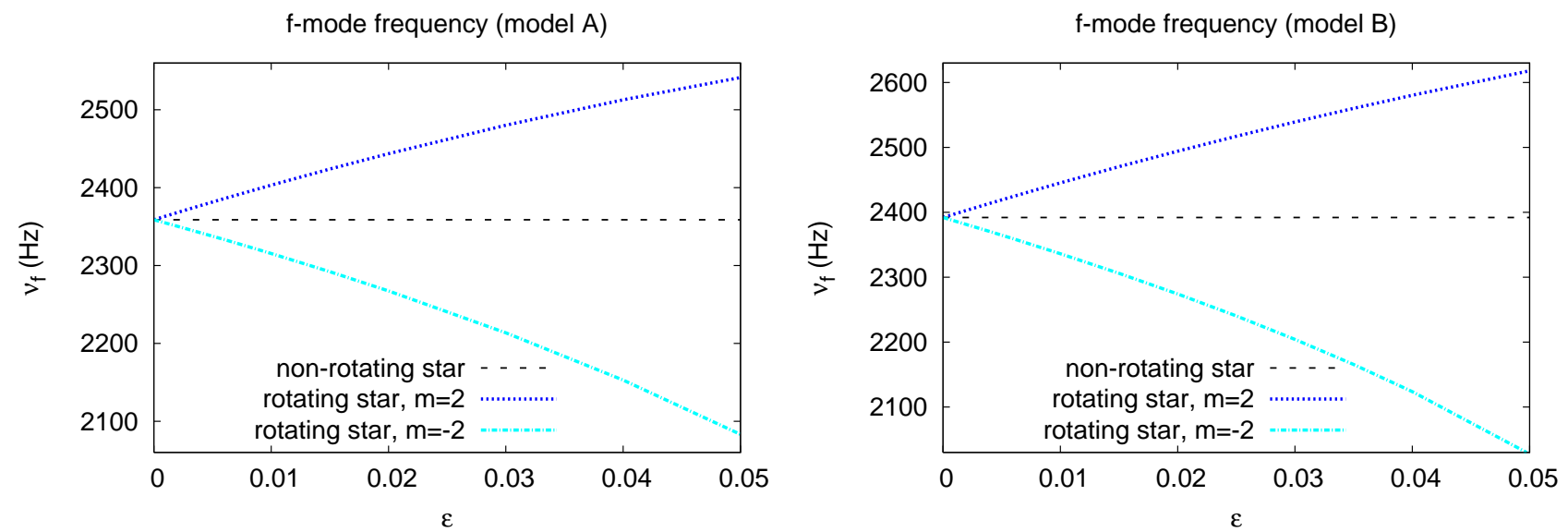

FIG. 3: The real part of the $f$-mode frequency, $\nu_{f}=\sigma_{f}^{R} /(2 \pi)$, is plotted versus the rotation parameter $\epsilon$. The data refer to two models of constant density star (see text). We include couplings up to $l=4$. The dashed line refers to the non rotating star; the dotted lines refer to the modes $m= \pm 2$ of the slowly rotating star.

Figure 4 shows, in a smaller range of the rotation parameter, the frequency $\nu_{f}$ computed by truncating the expansion in $l$ at $L=2$ (i.e. without couplings), $L=3$ and $L=4$. It is evident that for slowly rotating stars the contribution of the couplings is a small correction, and that there is convergence as $L$ grows.

The relative frequency shift due to the couplings is well approximated by a quadratic behaviour in $\epsilon$ :

$$
\frac{\Delta \nu_{f}}{\nu_{f}}=\frac{\nu_{f}^{L=4}(\epsilon)-\nu_{f}^{L=2}(\epsilon)}{\nu_{f}^{\epsilon=0}} \simeq \sigma^{\prime \prime} \epsilon^{2},
$$

as shown in Figure 5. Therefore, the contribution of the couplings is of order $O\left(\epsilon^{2}\right)$, as argued by Kojima in K2, and $\sigma_{f}^{R}$ is well described by a quadratic fit of the form

$$
\sigma_{f}^{R}=\sigma_{0}^{R}\left(1+m \epsilon \sigma_{R}^{\prime}+\epsilon^{2} \sigma_{R}^{\prime \prime}\right)
$$

where we should remind that terms of order $O\left(\epsilon^{2}\right)$ are of the same order of the terms which we are neglecting in the perturbed equations ab initio. This fit is accurate up to $\epsilon \sim 10^{-3}$; for larger values $\sigma^{\prime}$ and $\sigma^{\prime \prime}$ are no longer constant, as shown in Figure 6. as $\epsilon$ grows, $\sigma^{\prime}$ changes linearly, and the change is negative if $m>0$, positive if $m<0$, yielding in both cases a shift to lower values of the total frequency $\sigma$. This explains the small asymmetry between $\nu_{f}(m=2)$ and $\nu_{f}(m=-2)$ shown in Figure 3. Notice that deviations from the fit (61) are always of order $O\left(\epsilon^{2}\right)$, consistently 

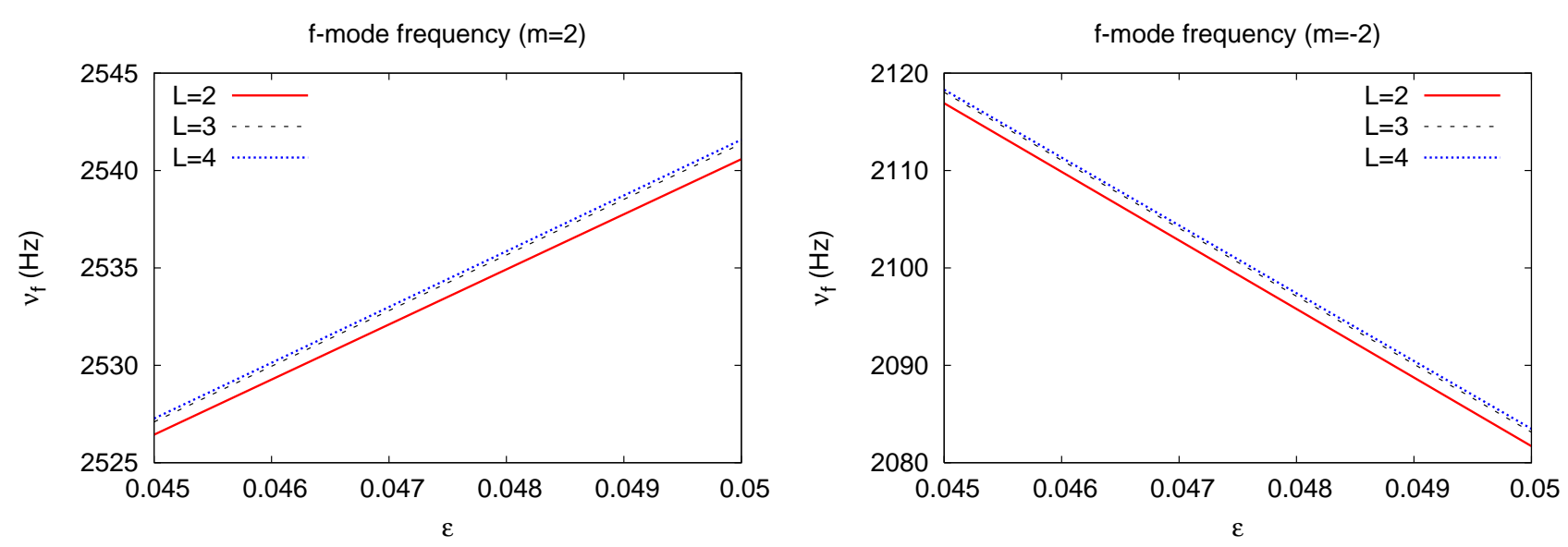

FIG. 4: Details of left panel of Figure 3 we show the contribution of the different couplings to the $f$-mode frequency, for model $A$. The different curves are obtained by including in the perturbed equations couplings up to $l=L$.

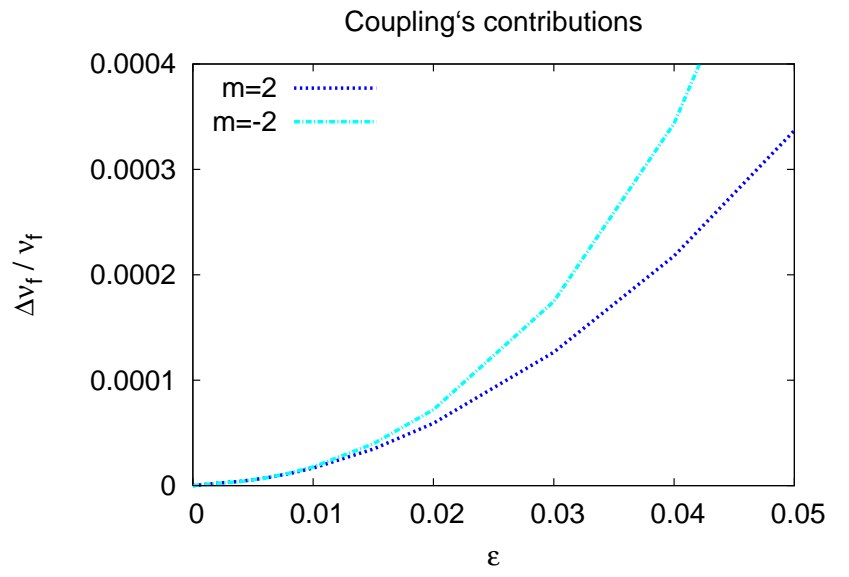

FIG. 5: Frequency shift as given by equation (60) for model A.

with our approximation scheme. For $0.01<\epsilon<0.05$, the coefficients $\sigma^{\prime \prime}$ are $\sim 0.15$ (if $m=2$ ) and $\sim 0.20$ (if $m=-2$ ); for $\epsilon<0.01$ they are too small to be correctly extrapolated with our codes. Finally, the damping time of the $f$-mode is shown in Figure 7 as a function of the rotation rate.

It is worth stressing that, as the stellar rotation increases, the frequency of the counterrotating (i.e. $m=-2$ ) mode decreases faster than expected by the simple linear fit (59). Furthermore, Figure 7 shows that the damping time of the counterrotating mode increases sharply, even for small rotation rates. This indicates that the CFS instability may occur for values of the rotation rate lower than expected by simple linear estimates.

It should be mentioned that the equations derived for the perturbations of a slowly rotating star in [7] and in Appendix $\mathrm{B}$, are not appropriate to study the $r$-modes, because the frequency $\sigma$, which is a dimensionful scale of the problem, is of the same order as the "small" parameter $\epsilon$. The shift of the $r$-mode frequency due to slow rotation in a relativistic star has been studied in [16], taking into account the couplings between perturbations with different $l$ 's.

\section{CONCLUDING REMARKS}

In this paper we propose a new approach to find the quasi-normal mode frequencies of rotating relativistic stars. We describe the main features of the general method, and test it in the particular case of slowly rotating stars. The application of our method to rapidly rotating stars will be discussed in a forthcoming paper.

We give explicit formulae (whose numerical implementation is straightforward) which allow to transform functions of $r, \theta$ in vectors, and systems of coupled differential equations (involving derivatives in $r, \theta$ ) in algebraic, matrix equations. Furthermore, we show that, once the 2D-equations describing stellar perturbations have been solved, the 
$\sigma^{\prime}(\mathrm{m}=2)$

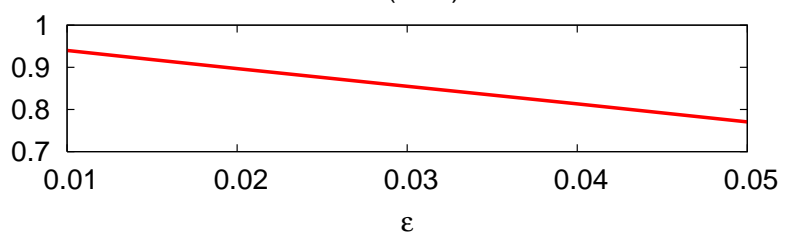

$\sigma^{\prime \prime}(m=2)$

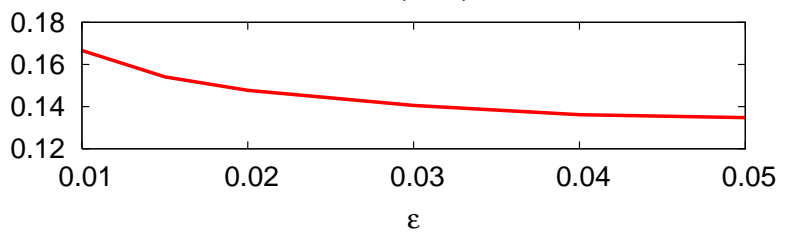

$\sigma^{\prime}(m=-2)$

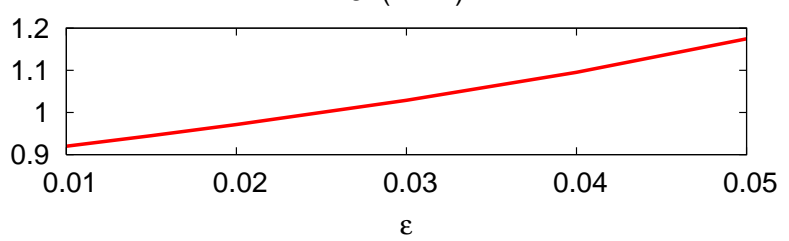

$\sigma^{\prime \prime}(m=-2)$

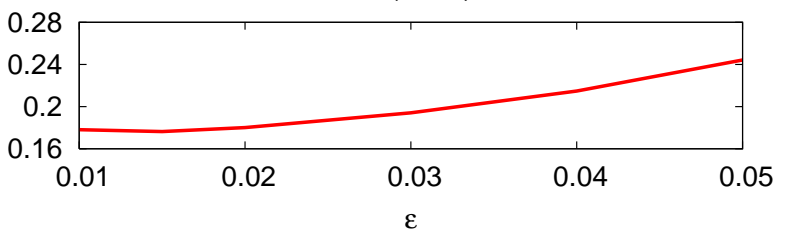

FIG. 6: The coefficients $\sigma^{\prime}, \sigma^{\prime \prime}$, plotted as functions of the rotation rate for model $A$.

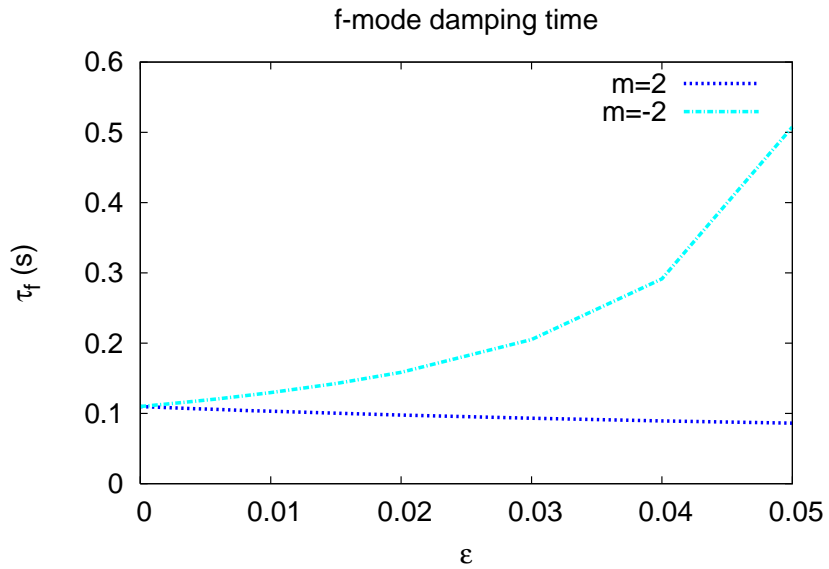

FIG. 7: Damping time of the $f$-mode as a function of the rotation rate, for model $A$.

frequencies and damping times of slowly damped, quasi-normal modes can be found by looking for the minima of the determinant of a properly defined matrix, evaluated as function of real frequency, thus generalizing the standing wave approach [23, 24] to rotating stars.

We have tested the method in the case of slow rotation; the system of partial differential equations from which we start is formally the same as in [7] (apart from a redefinition of some variables described in Appendix B). However, in our approach we transform that differential system in a system of algebraic, coupled equations; thus, the advantage of our method is that it is much easier to handle the couplings among different values of $l$, which in 7] correspond to couplings between different partial differential equations. For this reason we are able to study the shift of the fundamental mode due to rotation, taking into account the $l \leftrightarrow l \pm 1$ couplings, to our knowledge for the first time in the literature.

In this paper we show that, as the rotation parameter $\epsilon$ increases, the frequency of counterrotating modes decreases at a rate higher than linear in $\epsilon$. Furthermore, the corresponding damping time sharply increases, even for small rotation rates. This suggests that the CFS instability for a generic mode should occur for values of the rotation rate lower than expected by simple linear estimates. This result complements what found in [19], where the equations of stellar perturbations where integrated in full general relativity looking for neutral modes, and it was shown that the CFS instability sets in for smaller rotation rates than in Newtonian gravity.

It should be mentioned that an alternative approach to find the QNM frequencies, based on a characteristic formulation of the perturbed equations and a complexification of the radial coordinate, which could be generalized to rotating stars, has recently been proposed [34]. 


\section{Acknowledgments}

We are grateful to Kostas Kokkotas for suggesting us to generalize the standing wave approach to rotating stars. We thank Loic Villain for useful suggestions about the spectral methods, and Josè Pons for useful discussions. S.M. is supported by a "Virgo EGO Scientific Forum (VESF)" grant.

\section{APPENDIX A: GAUGE CHOICE}

In this Appendix we denote with $A, B, \ldots$ the coordinates $t, r$, and with $a, b, \ldots$ the coordinates $\theta, \phi$; we define $\gamma_{a b} \equiv \operatorname{diag}\left(1, \sin ^{2} \theta\right)$.

\section{The generalized Regge-Wheeler gauge}

There are many possible gauge choices to study the perturbations of a stationary, axisymmetric spacetime (see for instance [19]). Here we describe a particular gauge which has the property to reduce, when the background becomes spherically symmetric, to the well-known Regge-Wheeler gauge [28]. As discussed in Section II, we assume a background metric in the coordinates $(t, r, \theta, \phi)$ like (2) or (3), with $\frac{\partial}{\partial t}$, $\frac{\partial}{\partial \phi}$ Killing vectors. The metric perturbations have the form

$$
h_{\mu \nu}(t, r, \theta, \phi)=h_{\mu \nu}^{m \omega}(r, \theta) e^{\mathrm{i} m \phi} e^{-\mathrm{i} \omega t} .
$$

We shall show that it is possible to fix the gauge in such a way that the metric perturbation takes the form

$$
h_{\mu \nu}^{m \omega}(r, \theta)=\left(\begin{array}{cc|cc}
e^{\nu} H_{0}^{m \omega}(r, \theta) & H_{1}^{m \omega}(r, \theta) & -\frac{\mathrm{i} m}{\sin \theta} h_{0}^{m \omega}(r, \theta) & \begin{array}{r}
\sin \theta h_{0, \theta}^{m \omega}(r, \theta) \\
\sin \theta h_{1, \theta}^{m \omega}(r, \theta)
\end{array} \\
\hline \ldots & e^{\lambda} H_{2}^{m \omega}(r, \theta) & -\frac{i m}{\sin \theta} h_{1}^{m \omega}(r, \theta) & \sin (r, \theta) r^{2} \sin ^{2} \theta
\end{array}\right),
$$

and depends on six quantities

$$
\left[H_{0}^{m \omega}(r, \theta), H_{1}^{m \omega}(r, \theta), H_{2}^{m \omega}(r, \theta), K^{m \omega}(r, \theta), h_{0}^{m \omega}(r, \theta), h_{1}^{m \omega}(r, \theta)\right] .
$$

These quantities behave as scalars with respect to rotations. In order to fix the gauge (A2) we impose the following conditions

$$
\begin{aligned}
& h_{a b}^{m \omega}(r, \theta) \propto \gamma_{a b} \\
& \int_{0}^{\pi} d \theta \sin \theta\left[Y_{, \theta}^{l m}(\theta, 0) h_{A \theta}^{m \omega}(r, \theta)+\frac{\mathrm{i} m}{\sin ^{2} \theta} Y^{l m}(\theta, 0) h_{A \phi}^{m \omega}(r, \theta)\right]=0 \quad \forall l .
\end{aligned}
$$

These conditions correspond to setting to zero four functions of $(r, \theta)$ :

$$
\begin{aligned}
& h_{\theta \phi}^{m \omega}(r, \theta)=0, \\
& h_{\theta \theta}^{m \omega}(r, \theta)-\frac{1}{\sin ^{2} \theta} h_{\phi \phi}^{m \omega}(r, \theta)=0, \\
& \sum_{l=|m|}^{\infty} \int_{0}^{\pi} d \theta \sin \theta\left[Y_{, \theta}^{l m}(\theta, 0) h_{t \theta}^{m \omega}(r, \theta)+\frac{\mathrm{i} m}{\sin ^{2} \theta} Y^{l m}(\theta, 0) h_{t \phi}^{m \omega}(r, \theta)\right]=0, \\
& \sum_{l=|m|}^{\infty} \int_{0}^{\pi} d \theta \sin \theta\left[Y_{, \theta}^{l m}(\theta, 0) h_{r \theta}^{m \omega}(r, \theta)+\frac{\mathrm{i} m}{\sin ^{2} \theta} Y^{l m}(\theta, 0) h_{r \phi}^{m \omega}(r, \theta)\right]=0,
\end{aligned}
$$

and can be imposed through a diffeomorphism generated by the vector field

$$
\xi_{\mu}(t, r, \theta, \phi)=\xi_{\mu}^{m \omega}(r, \theta) e^{\mathrm{i} m \phi} e^{-\mathrm{i} \omega t},
$$

which depends on four functions of $(r, \theta)$. 
The relation between (A2) and (A4) is trivial, but to show that (A5) implies (A2) is less obvious. If we integrate by parts (A5), we find

$$
\int_{0}^{\pi} d \theta Y^{l m}(\theta, 0)\left[-\left(\sin \theta h_{A \theta}^{m \omega}(r, \theta)\right)_{, \theta}+\frac{\mathrm{i} m}{\sin \theta} h_{A \phi}^{m \omega}(r, \theta)\right]=0 .
$$

As it holds for all l's, the term in square brackets must vanish identically, i.e.

$$
-\left(\sin \theta h_{A \theta}^{m \omega}(r, \theta)\right)_{, \theta}+\frac{\mathrm{i} m}{\sin \theta} h_{A \phi}^{m \omega}(r, \theta) \equiv 0,
$$

therefore we can express the four quantities $\left(h_{A \theta}^{m \omega}(r, \theta), h_{A \phi}^{m \omega}(r, \theta)\right)$ in terms of two scalar functions $h_{A}^{m \omega}(r, \theta)$ such that:

$$
\begin{aligned}
& h_{A \theta}^{m \omega}(r, \theta)=-\frac{\mathrm{i} m}{\sin \theta} h_{A}^{m \omega}(r, \theta) \\
& h_{A \phi}^{m \omega}(r, \theta)=\left(\sin \theta h_{A}^{m \omega}(r, \theta)\right)_{, \theta} .
\end{aligned}
$$

This, together with (A4), gives A2 . This gauge choice is implicit in the formulation used in K1 to describe the perturbations of slowly rotating stars. It can, in principle, also be chosen to describe perturbations of rapidly rotating stars.

We stress that the existence of the generalized Regge-Wheeler gauge, in which all perturbations are expressed in terms of functions that are scalar with respect to rotation, is important because it provides a solid basis to the approach described in this paper. Indeed, it guarantees that expanding the perturbations in tensorial spherical harmonics is equivalent to expand in circular harmonics $e^{\mathrm{i} m \phi}$ firstly, and then to expand the functions appearing in the resulting equations in $(r, \theta)$, in associate Legendre polynomials, $P^{l m}(\theta)$.

\section{Relations with the Regge-Wheeler gauge}

In order to better understand how the gauge (A2) is related to the Regge-Wheeler (RW) gauge, we now expand $h_{\mu \nu}(t, r, \theta, \phi)$ in tensor spherical harmonics. This is always possible (on a surface $t=$ const., $r=$ const.), but typically it is not useful if the background is non-spherical, since the dynamical equations couple perturbations with different l's. Anyway, for slowly rotating stars the couplings are small, and the spherical harmonics expansion, as described in Appendix [B] and in [7], turns out to be useful.

By expanding the perturbed metric tensor in tensor spherical harmonics, before any gauge fixing we find

$$
\begin{aligned}
& h_{\mu \nu}(t, r, \theta, \phi)=h_{\mu \nu}^{m \omega}(r, \theta) e^{\mathrm{i} m \phi} e^{-\mathrm{i} \omega t}= \\
& \sum_{l}\left(\begin{array}{cc|c}
e^{\nu} H_{0}^{l m}(r) Y^{l m}(\theta, \phi) & H_{1}^{l m}(r) Y^{l m}(\theta, \phi) & h_{0, p o l}^{l m}(r) Y_{, a}^{l m}(\theta, \phi)+h_{0}^{l m}(r) S_{a}^{l m}(\theta, \phi) \\
\ldots & e^{\lambda} H_{2}^{l m}(r) Y^{l m}(\theta, \phi) & h_{1, p o l}^{l m}(r) Y_{, a}^{l m}(\theta, \phi)+h_{1}^{l m}(r) S_{a}^{l m}(\theta, \phi) \\
\hline \ldots & \ldots & K^{l m}(r) r^{2} \gamma_{a b} Y^{l m}(\theta, \phi)+G^{l m}(r) Z_{a b}^{l m}(\theta, \phi)+h_{a x}^{l m}(r) S_{a b}^{l m}(\theta, \phi)
\end{array}\right) e^{-\mathrm{i} \omega t}
\end{aligned}
$$

where

$$
S_{a}^{l m}(\theta, \phi)=\left(S_{\theta}^{l m}, S_{\phi}^{l m}\right)=\left(-\frac{1}{\sin \theta} Y_{, \phi}^{l m}, \sin \theta Y_{, \theta}^{l m}\right)
$$

are the axial vector harmonics, and $Z_{a b}$ and $S_{a b}$ are tensor harmonics satisfying $\gamma^{a b} Z_{a b}=\gamma^{a b} S_{a b}=0$, with polar and axial parity, respectively.

The RW-gauge for a spherical background, imposes [28]:

$$
h_{0, p o l}^{l m}=h_{1, p o l}^{l m}=h_{a x}^{l m}=G^{l m}=0 .
$$

If we consider (A13) in the case of a non-spherical background, we see that it is equivalent to the gauge (A2). Indeed, expanding $h_{A a}(t, r, \theta, \phi)$ in vector spherical harmonics, we find

$$
h_{A a}^{m \omega}(r, \theta) e^{\mathrm{i} m \phi}=\sum_{l}\left[h_{A p o l}^{l m}(r) Y_{, a}^{l m}(\theta, \phi)+h_{A a x}^{l m}(r) S_{a}^{l m}(\theta, \phi)\right],
$$


which, if $h_{A p o l}^{l m}=0$, reduces to

$$
\begin{aligned}
h_{A a}^{m \omega}(r, \theta) e^{\mathrm{i} m \phi} & =\sum_{l} h_{A a x}^{l m}(r) S_{a}^{l m}(\theta, \phi)=\sum_{l} h_{A a x}^{l m}(r)\left(-\frac{1}{\sin \theta} Y_{, \phi}^{l m}, \sin \theta Y_{, \theta}^{l m}\right) \\
& =\left(-\frac{\mathrm{i} m}{\sin \theta} h_{A}, \sin \theta h_{A, \theta}\right) e^{\mathrm{i} m \phi},
\end{aligned}
$$

where we have defined the scalar functions

$$
h_{A}(r, \theta) \equiv \sum_{l} h_{A a x}^{l m}(r) Y^{l m}(\theta, 0) .
$$

\section{The Regge-Wheeler equation for a spherical star as a 2D-equation in $r$ and $\theta$}

We conclude this section with a simple exercise. Choosing the gauge A2 , we derive the equations which describe the axial perturbations of a non rotating star, i.e. the equations for $h_{A}^{m \omega}(r, \theta)$ (in this case fluid perturbations are decoupled from metric perturbations), and we show how to solve them using spectral methods. We shall follow the lines of the well-known derivation of the Regge-Wheeler equation [28], with one difference: we do not expand the perturbations in spherical harmonics. By defining the function

$$
Z^{m \omega}(r, \theta) \equiv \frac{1}{r} h_{1}^{m \omega}(r, \theta) e^{(\nu-\lambda) / 2}
$$

from Einstein's equations we find

$$
h_{0}^{m \omega}(r, \theta)=\frac{1}{\mathrm{i} \sigma}\left(r Z^{m \omega}(r, \theta)\right)^{\prime} e^{(\nu-\lambda) / 2},
$$

where " indicates differentiation with respect to $r . Z^{m \omega}(r, \theta)$ satisfies the partial differential equation

$$
\partial_{r_{*}}^{2} Z^{m \omega}+\left(\sigma^{2}-V\right) Z^{m \omega}=0
$$

where the coordinate $r_{*}$ is defined by

$$
\frac{d r_{*}}{d r}=e^{(\lambda-\nu) / 2}
$$

In this formulation, $V$ is a differential operator:

$$
V \equiv \frac{e^{\nu}}{r^{2}}\left[-\left(\partial_{\theta}^{2}+\cot \theta \partial_{\theta}-\frac{m^{2}}{\sin ^{2} \theta}\right)-\frac{6 M}{r}+4 \pi(\rho-p) r^{2}\right]
$$

The usual one-dimensional Regge-Wheeler equation can easily be recovered by expanding $Z^{m \omega}(r, \theta)$ in scalar spherical harmonics:

$$
Z^{m \omega}(r, \theta)=\sum_{l=l_{m i n}}^{\infty} Z^{l m \omega}(r) Y^{l m}(\theta, 0) \rightarrow\left\{\begin{array}{l}
\frac{d^{2}}{d r_{*}^{2}} Z^{l m \omega}(r)+\left(\sigma^{2}-V^{l}\right) Z^{l m \omega}(r)=0 \\
V^{l}=\frac{e^{\nu}}{r^{2}}\left(l(l+1)-\frac{6 M}{r}+4 \pi(\rho-p) r^{2}\right)
\end{array}\right.
$$

where

$$
l_{\text {min }} \equiv \max (|m|, 2),
$$

and $Z^{\operatorname{lm} \omega}(r)$ is the standard Regge-Wheeler function.

Let us briefly describe how equation (A19) can be integrated using spectral methods and the standing wave approach. We shall integrate this equation for real values of the frequency, therefore in the following we shall set $\omega=\sigma$. The integration range in $r_{*}$ is $r_{*}=\left[r_{* 1}, r_{* 2}\right]$, i.e. given $Z^{m \sigma}\left(r_{* 1}, \theta\right)$ we want to know $Z^{m \sigma}\left(r_{* 2}, \theta\right)$. The starting point $r_{* 1}$ corresponds to a small sphere near the center of the star, with $r=r_{0} \ll R$, where the Regge-Wheeler function is given by an analytical expansion (see below). The final point, $r_{* 2}$, corresponds to a point in the wave zone, $r=r_{\infty} \gg R$, where the ingoing and outgoing amplitudes can be extracted. 
We rescale the variables $r_{*}$ and $\theta$ as follows:

$$
\begin{aligned}
& x=\frac{2 r_{*}-r_{* 1}-r_{* 2}}{r_{* 1}-r_{* 2}} \in[-1,1] \\
& y=\cos \theta \in[-1,1],
\end{aligned}
$$

so that $r_{* 1}$ corresponds to $x=1$, and $r_{* 2}$ to $x=-1$. Then, we perform the double expansion (53) of the Regge-Wheeler function $Z^{m \sigma}(r, \theta)$ defined in (A17), for assigned values of $m, \sigma$ :

$$
Z^{m \sigma}(x, y)=\sum_{n=0}^{K} \sum_{l=l_{m i n}}^{L} a_{n}^{l m} \sigma T_{n}(x) P^{l m}(y) .
$$

The expansions in Chebyshev's and associated Legendre's polynomials are truncated at $K$ and $L$, respectively (for instance, $L=10, K=20$ ). The boundary conditions at the center of the star are imposed by assigning $Z^{m \sigma}$ and its derivative at $x=1$ :

$$
\begin{aligned}
Z^{m \sigma}(x=1, y) & =\sum_{l=l_{\min }}^{L} z_{0}^{l} \hat{H}^{l m} P^{l m}(y) \\
\partial_{r_{*}} Z^{m \sigma}(x=1, y) & =\sum_{l=l_{\min }}^{L} z_{1}^{l} \hat{H}^{l m} P^{l m}(y),
\end{aligned}
$$

where the analytic expansion of the Regge-Wheeler equation gives [3]

$$
\begin{aligned}
z_{0}^{l} & =r_{0}^{l+1}+X_{i n}^{l}(\sigma) r_{0}^{l+3} \\
z_{1}^{l} & =e^{\nu(0)}\left[(l+1) r_{0}^{l}+(l+3) X_{i n}^{l}(\sigma) r_{0}^{l+2}\right] \\
X_{i n}^{l}(\sigma) & \equiv \frac{(l+2)\left[\frac{1}{3}(2 l-1) \rho(0)-p(0)\right]-\sigma^{2} e^{\nu(0)}}{2(2 l+3)}
\end{aligned}
$$

(note that while in $z_{0,1}^{l}$ and $X_{i n}^{l}$, the index $l$ is a superscript, in $r_{0}^{l}$ it is an exponent).

The constants $\hat{H}^{l m}$ form a vector

$$
\hat{\mathbf{H}}^{m} \equiv\left(\hat{H}^{l m}\right)
$$

which can be freely assigned; for each vector $\hat{\mathbf{H}}$ we have one solution of the equation. Notice that we have one constant $\hat{H}^{l m}$ for each value of $l$ i.e., in the language of Section IIA2, $N=1$ for the axial parity perturbations. If, in addition to axial perturbations, polar parity perturbations are considered, described outside the star by the Zerilli equation, then there is another constant to be assigned for each value of $l$. Therefore, if all metric perturbations are considered, $N=2$ as discussed in Section $\amalg A 2$.

We now project equation (A19) in the basis of Chebyshev's and associate Legendre's polynomials. The operator

$$
\partial_{\theta}^{2}+\cot \theta \partial_{\theta}-\frac{m^{2}}{\sin ^{2} \theta}
$$

is diagonal in the $P^{l m}$ basis, with eigenvalue $-l(l+1)$. Therefore, in this basis the operator $V$ defined in (A21) reduces to the one-dimensional Regge-Wheeler potential $V^{l}(r)$ (A22). We introduce (as in in Section IIB 1) Equations (31), (34) ) the derivative matrix $D_{n n^{\prime}}$ and the potential matrix $V_{n m}$ obtained projecting $V^{l}(r)$ on Chebyshev polynomials. To write the matrix equation we define the collective index

$$
i(l, n)=(l-|m|)(K+1)+n+1 \in[1, \mathcal{N}]
$$

with $\mathcal{N}=(L-|m|+1)(K+1)$, and we define the $\mathcal{N}$-dimensional vector of components

$$
\hat{a}_{i}^{m}=\hat{a}_{i(l, n)}^{m}=a_{n}^{l m} .
$$

The matrix equation has the block-diagonal form

$$
\hat{L}_{i i^{\prime}} a_{i^{\prime}}^{m}=0
$$


with

$$
\hat{L}=\left(\begin{array}{ccc}
\left(D_{n n^{\prime}}^{2}+\tilde{V}_{n n^{\prime}}\right)_{l=|m|} & 0 & \\
& \left(D_{n n^{\prime}}^{2}+\tilde{V}_{n n^{\prime}}\right)_{l=|m|+1} & \\
& \ddots & \\
0 & & \left(D_{l l^{\prime}}^{2}+\tilde{V}_{n n^{\prime}}\right)_{l=L}
\end{array}\right) .
$$

The boundary conditions are implemented by replacing the last two lines of each block, i.e. each $i(l, K)$-th and $i(l, K-1)$-th lines, with the conditions at the center in terms of the vector $\hat{\mathbf{H}}^{m}=\left(\hat{H}^{l m}\right)$ :

$$
\begin{aligned}
\sum_{n=0}^{K} \hat{a}_{i(l, n)}^{m} & =z_{0}^{l} \hat{H}^{l m} \\
\sum_{n, k}^{0, K} \hat{D}_{k n} \hat{a}_{i(l, n)}^{m} & =z_{1}^{l} \hat{H}^{l m} .
\end{aligned}
$$

By inverting the matrix equation (A32) we find the coefficients $\hat{a}_{i}^{m}$ and then $Z^{m \omega}(-1, y)$.

In this way, for each choice of $(m, \sigma)$, and of the vector $\hat{\mathbf{H}}^{m}$ of initial conditions, we can integrate from $r_{0}$ to $r_{\infty}$. The ingoing amplitude at infinity

$$
\mathcal{A}^{m}(\sigma, \theta)=\sum_{l} \mathcal{A}^{l m}(\sigma) P^{l m}(\theta) .
$$

can be found using the algorithm described in [24]. Therefore, for each choice of the vector of initial conditions $\hat{\mathbf{H}}^{m}=\left(\hat{H}^{l m}\right)$, we have the vector of ingoing amplitudes at infinity

$$
\mathbf{A}^{m}=\left(\mathcal{A}^{l m}(\sigma)\right)
$$

This procedure is linear at any step, thus, repeating it for all $\hat{\mathbf{H}}^{m}$ :

$$
\hat{\mathbf{H}}^{m}=(1,0, \ldots, 0), \quad \hat{\mathbf{H}}^{m}=(0,1, \ldots, 0), \quad \ldots, \quad \hat{\mathbf{H}}^{m}=(0,0, \ldots, 1)
$$

we find the expression of the matrix $\mathbf{M}^{m}=\left(\mathcal{M}^{m \mid l l^{\prime}}(\sigma)\right)$ defined by

$$
\mathcal{A}^{l m}(\sigma)=\mathcal{M}^{m \mid l l^{\prime}}(\sigma) \hat{H}^{l^{\prime} m}
$$

As explained in Section II 2 near a mode $\sigma \sim \sigma_{0}$ the modulus of the determinant of this matrix behaves as

$$
\left|\operatorname{det} \mathbf{M}^{m}(\sigma)\right| \propto \sqrt{\left(\sigma-\sigma_{0}\right)^{2}+{\frac{1}{\tau_{0}}}^{2}}
$$

and the frequency $\sigma_{0}$ and the damping time $\tau_{0}$ of the mode can be found by a quadratic fit in $\sigma$.

\section{APPENDIX B: EQUATIONS FOR THE PERTURBATIONS OF SLOWLY ROTATING STARS}

This derivation of the equations describing the perturbations of a slowly rotating star is based on the work of Kojima [7], denoted as K1. As discussed above, since we are considering slowly rotating stars, we can first expand the perturbations in spherical harmonics, getting a system of coupled ODE in $r$, and then expand this system in Chebyshev polynomials, getting an algebraic matrix equation. The first step of this program is equivalent to the derivation of K1, with one difference: we are going to reformulate the equations in terms of a different set of variables, which are numerically well behaved near the center of the star. Following K1, we shall assume $l \geq 2$.

The background configuration, describing a slowly rotating, stationary and axially symmetric star is given by equation (56). The pressure $p$ and the energy density $\rho$ are found by solving the TOV equations; we assume that the 
equation of state of matter in the star is barotropic, $p=p(\rho)$; therefore, $c_{s}^{2} \equiv\left(\frac{\partial p}{\partial \rho}\right)=\frac{p^{\prime}}{\rho^{\prime}}$. The perturbations of the background (56) can be written as

$$
\begin{aligned}
& h_{\mu \nu}=\sum_{l m}\left(\begin{array}{cc|cc}
e^{\nu} H_{0}^{l m}(r) Y^{l m}(\theta, \phi) H_{1}^{l m}(r) Y^{l m}(\theta, \phi) & h_{0}^{l m}(r) S_{\theta}^{l m}(\theta, \phi) & h_{0}^{l m}(r) S_{\phi}^{l m}(\theta, \phi) \\
\ldots & e^{\lambda} H_{2}^{l m}(r) Y^{l m}(\theta, \phi) & h_{1}^{l m}(r) S_{\theta}^{l m}(\theta, \phi) & h_{1}^{l m}(r) S_{\phi}^{l m}(\theta, \phi) \\
\hline \ldots & \ldots & K^{l m}(r) r^{2} Y^{l m}(\theta, \phi) & 0 \\
\ldots & \ldots & K^{l m}(r) r^{2} \sin ^{2} \theta Y^{l m}(\theta, \phi)
\end{array}\right) e^{-\mathrm{i} \sigma t} \\
& \delta u^{r}=\frac{e^{\nu / 2-\lambda}}{4 \pi(\rho+p)} R^{l m}(r) Y^{l m}(\theta, \phi) e^{-\mathrm{i} \sigma t} \\
& \delta u^{\theta}=\frac{e^{\nu / 2-\lambda}}{4 \pi(\rho+p)}\left(V^{l m}(r) Y_{, \theta}^{l m}(\theta, \phi)+U^{l m}(r) S_{\theta}^{l m}(\theta, \phi)\right) e^{-\mathrm{i} \sigma t} \\
& \delta u^{\phi}=\frac{e^{\nu / 2-\lambda}}{4 \pi(\rho+p)}\left(V^{l m}(r) Y_{, \phi}^{l m}(\theta, \phi)+U^{l m}(r) S_{\phi}^{l m}(\theta, \phi)\right) e^{-\mathrm{i} \sigma t} \\
& \delta \rho=\delta \rho^{l m}(r) Y^{l m}(\theta, \phi) e^{-\mathrm{i} \sigma t} \\
& \delta p=\delta p^{l m}(r) Y^{l m}(\theta, \phi) e^{-\mathrm{i} \sigma t} \text {, }
\end{aligned}
$$

with $\sigma$ real. The linearized Einstein equations for the radial part of these quantities are ordinary differential equations in $r$. As explained in Section III, the general structure of these equations is:

$$
\begin{aligned}
\mathcal{L}^{p o l}\left[H_{0}^{l m}, K^{l m} ; \sigma\right] & =m \mathcal{E}\left[H_{0}^{l m}, K^{l m} ; \sigma\right]+\mathcal{F}^{( \pm)}\left[Z_{R W}^{l \pm 1 m} ; \sigma\right] \\
\mathcal{L}^{a x}\left[Z_{R W}^{l m} ; \sigma\right] & =m \mathcal{N}\left[Z_{R W}^{l m} ; \sigma\right]+\mathcal{D}^{( \pm)}\left[H_{0}^{l \pm 1 m}, K^{l \pm 1 m} ; \sigma\right] .
\end{aligned}
$$

The quantities $H_{1}^{l m}, H_{2}^{l m}, R^{l m}, V^{l m}, U^{l m}, \delta \rho^{l m}, \delta p^{l m}$ can be expressed in terms of $H_{0}^{l m}, K^{l m}, Z_{R W}^{l m}$, once equations (B2) have been solved.

\section{The $O\left(\epsilon^{0}\right)$ equations}

The equations at $O\left(\epsilon^{0}\right)$ describe the perturbations of a non-rotating star.

The equations for polar perturbations inside the star are a system of two second order ODE in $K^{l m}, H_{0}^{l m}$ :

$$
\begin{aligned}
L_{\text {int }}^{(1) l m}\left[H_{0}, K\right] \equiv & \left(K^{l m}-H_{0}^{l m}\right)^{\prime \prime}-\frac{e^{\lambda}}{r^{2}}\left[2 r-10 M+4 \pi(\rho-5 p) r^{3}\right]\left(K^{l m}-H_{0}^{l m}\right)^{\prime} \\
& +\frac{e^{\lambda}}{r^{2}}\left(\sigma^{2} e^{-\nu} r^{2}-2 n\right)\left(K^{l m}-H_{0}^{l m}\right) \\
& +4 \frac{e^{\lambda}}{r^{4}}\left[3 M r-4 \pi \rho r^{4}-e^{\lambda}\left(M+4 \pi p r^{3}\right)^{2}\right] H_{0}^{l m}=0 \\
L_{\text {int }}^{(2) l m}\left[H_{0}, K\right] \equiv & K^{l m^{\prime \prime}}-\frac{e^{\lambda}}{r^{2}}\left[\left(r-3 M-4 \pi p r^{3}\right) c_{s}^{-2}-3 r+5 M+4 \pi \rho r^{3}\right] K^{l m^{\prime}} \\
& +\frac{e^{\lambda}}{r^{2}}\left[\sigma^{2} e^{-\nu} r^{2} c_{s}^{-2}-n\left(c_{s}^{-2}+1\right)\right] K^{l m}+\frac{c_{s}^{-2}-1}{r} H_{0}^{l m}{ }^{\prime} \\
& +\frac{e^{\lambda}}{r^{3}}\left[\left(n r+4 M+8 \pi p r^{3}\right) c_{s}^{-2}-(n+2) r+8 \pi \rho r^{3}\right] H_{0}^{l m}=0 .
\end{aligned}
$$

Once $H_{0}^{l m}, K^{l m}$ have been determined, $H_{1}^{l m}$ and $H_{2}^{l m}$ can be computed through the following relations:

$$
\begin{aligned}
& H_{1}^{l m}=-\frac{e^{\nu}}{\mathrm{i} \sigma}\left(H_{0}^{l m^{\prime}}-K^{l m^{\prime}}+\frac{2 e^{\lambda}}{r^{2}}\left(M+4 \pi p r^{3}\right) H_{0}^{l m}\right) \\
& H_{2}^{l m}=H_{0}^{l m} .
\end{aligned}
$$

Other relations (see K1) give the fluid perturbations $R^{l m}, V^{l m}, U^{l m}, \delta \rho^{l m}, \delta p^{l m}$ in terms of the metric perturbations. 
Outside the star, the polar perturbations reduce to a system of three first order ODE in $K^{l m}, H_{1}^{l m}$ and $H_{0}^{l m}$ :

$$
\begin{aligned}
L_{\text {ext }}^{(1) l m}\left[H_{0}, \tilde{H}_{1}, K\right] \equiv & K^{l m^{\prime}}+\frac{e^{\lambda}(r-3 M)}{r^{2}} K^{l m}-\frac{1}{r} H_{0}^{l m}-\frac{n+1}{r} \tilde{H}_{1}^{l m}=0 \\
L_{e x t}^{(2) l m}\left[H_{0}, \tilde{H}_{1}, K\right] \equiv & -r \tilde{H}_{1}^{l m^{\prime}}+e^{\lambda}\left(K^{l m}+H_{0}^{l m}\right)-\frac{2 M e^{\lambda}}{r} \tilde{H}_{1}^{l m}=0 \\
L_{e x t}^{(3) l m}\left[H_{0}, \tilde{H}_{1}, K\right] \equiv & H_{0}^{l m^{\prime}}+\frac{e^{\lambda}(r-3 M)}{r^{2}} K^{l m}-\frac{e^{\lambda}(r-4 M)}{r^{2}} H_{0}^{l m} \\
& +\left(\sigma^{2} r e^{\lambda}-\frac{n+1}{r}\right) \tilde{H}_{1}^{l m}=0,
\end{aligned}
$$

where we have defined

$$
\tilde{H}_{1}^{l m} \equiv-\frac{H_{1}^{l m}}{\mathrm{i} \sigma r}
$$

in order to have equations with real coefficients. Furthermore, there is an algebraic constraint:

$$
\begin{aligned}
L_{\text {ext }}^{(4) l m}\left[H_{0}, \tilde{H}_{1}, K\right] \equiv & \left(\sigma^{2} r^{4} e^{\lambda}-n r^{2}-M r+M^{2} e^{\lambda}\right) K^{l m} \\
& +(n r+3 M) r H_{0}^{l m}-\left[\sigma^{2} r^{4}-(n+1) M r\right] \tilde{H}_{1}^{l m}=0 .
\end{aligned}
$$

Equations (B6)-(B8) are equivalent to the Zerilli equation, but they have the advantage to be easily generalizable to rotating stars, as we will see below. The Zerilli function $Z_{Z \text { er }}$ can be computed in terms of $K, \tilde{H}_{1}$ :

$$
Z_{Z e r}^{l m}=\frac{r^{2}}{n r+3 M}\left(K^{l m}-e^{\nu} \tilde{H}_{1}^{l m}\right) .
$$

The axial perturbations are described by the Regge-Wheeler equation

$$
L^{l m}\left[Z_{R W}\right] \equiv \frac{d^{2}}{d r_{*}^{2}} Z_{R W}^{l m}+\left[\sigma^{2}-e^{\nu}\left(\frac{l(l+1)}{r^{2}}-\frac{6 M}{r^{3}}+4 \pi(\rho-p)\right)\right] Z_{R W}^{l m}=0,
$$

where the coordinate $r_{*}$ has been defined in (A20) and

$$
\begin{aligned}
& h_{0}^{l m}=-\frac{e^{(\nu-\lambda) / 2}}{\mathrm{i} \sigma}\left(Z_{R W}^{l m} r\right)^{\prime} \\
& h_{1}^{l m}=e^{(\lambda-\nu) / 2} Z_{R W}^{l m} r .
\end{aligned}
$$

An analytical expansion of equations (B3), (B4), (B12) near the center of the star gives, for each value of $l$, three independent conditions at the center:

$$
\begin{aligned}
K^{l m}-H_{0}^{l m} & =r^{l+2}+k h^{l m(+)} r^{l+4} \\
K^{l m} & =r^{l}+k^{l m(+)} r^{l+2} \\
Z_{R W}^{l m} & =0 \\
K^{l m}-H_{0}^{l m} & =r^{l+2}+k h^{l m(-)} r^{l+4} \\
K^{l m} & =-r^{l}+k^{l m(-)} r^{l+2} \\
Z_{R W}^{l m} & =0 \\
K^{l m}-H_{0}^{l m} & =0 \\
K^{l m} & =0 \\
Z_{R W}^{l m} & =r^{l+1}+z^{l m} r^{l+3}
\end{aligned}
$$

where the expressions for $k h^{l m( \pm)}, k^{l m( \pm)}, z^{l m}$ can be evaluated from the analytical expansion.

We notice that $K^{l m}$ and $H_{0}^{l m}$ behave, as $r \rightarrow 0$, like $r^{l}$, while the combination $K^{l m}-H_{0}^{l m}$ behaves as $r^{l+2}$. Consequently, when we expand $K^{l m}$ and $H_{0}^{l m}$ in powers of $r$ about $r=0$, we find that the leading terms are 
coincident. In other words, the differential equations for the variables $K^{l m}, H_{0}^{l m}$ and $Z_{R W}^{l m}$ are linearly dependent near the origin (see the discussion in [3] ). To avoid this problem, we use as integration variables $K^{l m}-H_{0}^{l m}, K^{l m}$ and $Z_{R W}^{l m}$.

Finally, we notice that at order $O\left(\epsilon^{0}\right)$, i.e. for a non rotating star, equation (B5) establishes that $H_{2}^{l m}=H_{0}^{l m}$. Therefore it is equivalent to use either $H_{0}^{l m}$ or $H_{2}^{l m}$.

\section{The $O\left(\epsilon^{1}\right)$ equations}

We define

$$
\begin{array}{cc}
n_{+} \equiv \frac{l(l+3)}{2} & n_{-} \equiv \frac{(l-2)(l+1)}{2} \\
Q_{l-1 m} \equiv \sqrt{\frac{(l-m)(l+m)}{(2 l-1)(2 l+1)}} & Q_{l+1 m} \equiv \sqrt{\frac{(l+1-m)(l+1+m)}{(2 l+1)(2 l+3)}} .
\end{array}
$$

The perturbed equations inside the star have the form

$$
\begin{aligned}
L_{\text {int }}^{(J) l m}\left[H_{0}, K\right]= & -\frac{m}{(n+1) \sigma} E^{(J) l m}\left[H_{0}, K\right] \\
& +e^{-(\lambda+\nu) / 2}\left[\frac{\mathrm{i} Q_{l-1 m} D^{(J) l-1 m}\left[Z_{R W}\right]}{\sigma\left(n-n_{-}\right)}+\frac{\mathrm{i} Q_{l+1 m} D^{(J) l+1 m}\left[Z_{R W}\right]}{\sigma\left(n-n_{+}\right)}\right] \\
L^{l m}\left[Z_{R W}\right]= & \frac{m}{\sigma} N^{l m}\left[Z_{R W}\right]+e^{(\lambda+3 \nu) / 2}\left[\frac{\mathrm{i} Q_{l-1 m} F^{l-1 m}\left[H_{0}, K\right]}{\sigma\left(n-n_{-}\right)}+\frac{\mathrm{i} Q_{l+1 m} F^{l+1 m}\left[H_{0}, K\right]}{\sigma\left(n-n_{+}\right)}\right]
\end{aligned}
$$

$(J=1,2)$, where $L_{i n t}^{(J) l m}\left[H_{0}, K\right], L^{l m}\left[Z_{R W}\right]$ are the operators defined in equations (B3), (B4), (B12) and $E^{(J) l m}\left[H_{0}, K\right]$, $D^{(J) l \pm 1 m}\left[Z_{R W}\right], N^{l m}\left[Z_{R W}\right], F^{l \pm 1 m}\left[H_{0}, K\right]$ are operators at first order in $\epsilon$, whose explicit expressions are given in K1. The operators $D^{(J) l \pm 1 m}\left[Z_{R W}\right], F^{l \pm 1 m}\left[H_{0}, K\right]$ couple perturbations belonging to different $l$ 's, and were neglected in the numerical integration of $\mathrm{K} 2$.

In order to have equations with real coefficients, we need to get rid of the factors i in (B18). To this purpose, we redefine the Regge-Wheeler function by a factor $-\mathrm{i}$

$$
Z_{R W}^{l m} \Rightarrow-\mathrm{i} Z_{R W}^{l m}
$$

thus equations (B18) become

$$
\begin{aligned}
L_{\text {int }}^{(J) l m}\left[H_{0}, K\right]= & -\frac{m}{(n+1) \sigma} E^{(J) l m}\left[H_{0}, K\right] \\
& +e^{-(\lambda+\nu) / 2}\left[\frac{Q_{l-1 m} D^{(J) l-1 m}\left[Z_{R W}\right]}{\sigma\left(n-n_{-}\right)}+\frac{Q_{l+1 m} D^{(J) l+1 m}\left[Z_{R W}\right]}{\sigma\left(n-n_{+}\right)}\right] \\
L^{l m}\left[Z_{R W}\right]= & \frac{m}{\sigma} N^{l m}\left[Z_{R W}\right]-e^{(\lambda+3 \nu) / 2}\left[\frac{Q_{l-1 m} F^{l-1 m}\left[H_{0}, K\right]}{\sigma\left(n-n_{-}\right)}+\frac{Q_{l+1 m} F^{l+1 m}\left[H_{0}, K\right]}{\sigma\left(n-n_{+}\right)}\right] .
\end{aligned}
$$

This rescaling consistently eliminates all imaginary units from the equations.

The numerical integration of equations (B20) presents a serious problem. If we perform an analytical expansion near the center of (B20), we find that the coupling terms $D^{(J) l-1 m}\left[Z_{R W}^{l m}\right]$ become larger than $L_{i n t}^{(J) l m}\left[H_{0}^{l m}, K^{l m}\right]$ as $r \rightarrow 0$. The reason behind this pathological behaviour is that near the center of the star

$$
\begin{aligned}
K^{l m}-H_{0}^{l m} & \sim r^{l+2}+(O(\epsilon) \text { terms }) \cdot r^{l} \\
K^{l m} & \sim r^{l} \\
H_{0}^{l m} & \sim r^{l} .
\end{aligned}
$$

If we use as integration variable $H_{2}^{l m}$ instead of $H_{0}^{l m}$ (notice that while in the non rotating case $H_{0}^{l m}=H_{2}^{l m}$, if the star rotates $\left.H_{0}^{l m}=H_{2}^{l m}+O(\epsilon)\right)$ this problem is overcome, since

$$
K^{l m}-H_{2}^{l m} \sim r^{l+2} .
$$


Consequently, the coupling terms in the perturbed equations are smaller than $L_{\text {int }}^{(J) l m}\left[H_{2}^{l m}, K^{l m}\right]$. For this reason, we have expressed our equations inside the star in terms of $K^{l m}-H_{2}^{l m}, K^{l m}, Z_{R W}^{l m}$ :

$$
\begin{aligned}
L_{\text {int }}^{(J) l m}\left[H_{2}, K\right]= & -\frac{m}{(n+1) \sigma} \tilde{E}^{(J) l m}\left[H_{2}, K\right] \\
& +e^{-(\lambda+\nu) / 2}\left[\frac{Q_{l-1 m} \tilde{D}^{(J) l-1 m}\left[Z_{R W}\right]}{\sigma\left(n-n_{-}\right)}+\frac{Q_{l+1 m} \tilde{D}^{(J) l+1 m}\left[Z_{R W}\right]}{\sigma\left(n-n_{+}\right)}\right] \\
L^{l m}\left[Z_{R W}\right]= & \frac{m}{\sigma} N^{l m}\left[Z_{R W}\right]-e^{(\lambda+3 \nu) / 2}\left[\frac{Q_{l-1 m} F^{l-1 m}\left[H_{2}, K\right]}{\sigma\left(n-n_{-}\right)}+\frac{Q_{l+1 m} F^{l+1 m}\left[H_{2}, K\right]}{\sigma\left(n-n_{+}\right)}\right] .
\end{aligned}
$$

The operators $\tilde{E}^{(J) l m}, \tilde{D}^{(J) l \pm 1 m}$ are different from $E^{(J) l m}, D^{(J) l \pm 1 m}$ given in K1. Their expressions are the following:

$$
\begin{aligned}
& \tilde{E}^{(1) l m}\left[H_{2}, K\right]=\frac{\mathrm{i} \sigma}{2}\left[2 f^{l m \prime \prime}(r)+\left(4 \nu^{\prime}-\lambda^{\prime}-\frac{6}{r}\right) f^{l m \prime}(r)\right. \\
& \left.-\left(\frac{2 e^{\lambda}}{r^{2}}(n+1)-\left(\nu^{\prime}\right)^{2}+\frac{4}{r}\left(2 \nu^{\prime}-\lambda^{\prime}-\frac{2}{r}\right)-32 \pi p e^{\lambda}\right) f^{l m}(r)\right] \\
& +\mathrm{i} \sigma\left(2 n \xi^{(1) l m \prime}(r)-\tilde{\beta}^{(1) l m \prime}(r)-\zeta^{(1) l m \prime}(r)\right) \\
& -\frac{\mathrm{i} \sigma}{2}\left(\frac{2}{r}+\lambda^{\prime}-2 \nu^{\prime}\right)\left(2 n \xi^{(1) l m}(r)-\tilde{\beta}^{(1) l m}(r)-\zeta^{(1) l m}(r)\right) \\
& +\mathrm{i} \sigma(n+1) e^{\lambda} C^{(3) l m}(r)-\mathrm{i} \sigma(n+1) C^{(2) l m}(r) \\
& \tilde{E}^{(2) l m}\left[H_{2}, K\right]=-\frac{\mathrm{i} \sigma}{r} c_{s}^{-2}\left[f^{l m \prime}(r)+\left(\nu^{\prime}-\frac{2}{r}+(n+1) \frac{e^{\lambda}}{r}\right) f^{l m}(r)\right] \\
& -\frac{\mathrm{i} \sigma}{r} c_{s}^{-2}\left(2 n \xi^{(1) l m}(r)-\tilde{\beta}^{(1) l m}(r)-\zeta^{(1) l m}(r)\right) \\
& -\frac{\mathrm{i} \sigma}{2}(n+1) c_{s}^{-2} C^{(2) l m}(r)+\frac{\mathrm{i} \sigma}{2}(n+1) e^{\lambda-\nu} C^{(0) l m}(r) \\
& \tilde{D}^{(1) l \pm 1 m}\left[Z_{R W}\right]=\mathrm{i} \sigma e^{(\lambda+\nu) / 2}\left[2 g^{l \pm 1 m \prime \prime}(r)+\left(4 \nu^{\prime}-\lambda^{\prime}-\frac{6}{r}\right) g^{l \pm 1 m^{\prime}}(r)\right. \\
& \left.-\left(\frac{2 e^{\lambda}}{r^{2}}(n+1)-\left(\nu^{\prime}\right)^{2}+\frac{4}{r}\left(2 \nu^{\prime}-\lambda^{\prime}-\frac{2}{r}\right)-32 \pi p e^{\lambda}\right) g^{l \pm 1 m}(r)\right] \\
& +2 \mathrm{i} \sigma e^{(\lambda+\nu) / 2}\left[-2\left(n-2 n_{ \pm}-2\right) \chi^{(1) l \pm 1 m^{\prime}}(r)+\left(n-n_{ \pm}-1\right) \tilde{\alpha}^{(1) l \pm 1 m^{\prime}}(r)-\eta^{(1) l \pm 1 m^{\prime}}(r)\right] \\
& -\mathrm{i} \sigma e^{(\lambda+\nu) / 2}\left(\frac{2}{r}+\lambda^{\prime}-2 \nu^{\prime}\right)\left[-2\left(n-2 n_{ \pm}-2\right) \chi^{(1) l \pm 1 m}(r)\right. \\
& \left.+\left(n-n_{ \pm}-1\right) \tilde{\alpha}^{(1) l \pm 1 m}(r)-\eta^{(1) l \pm 1 m}(r)\right] \\
& +\mathrm{i} \sigma e^{(3 \lambda+\nu) / 2}\left[\left(n-n_{ \pm}\right) \tilde{A}^{(3) l \pm 1 m}(r)+\left(n-n_{ \pm}\right)\left(n-n_{ \pm}-1\right) B^{(3) l \pm 1 m}(r)\right] \\
& -\mathrm{i} \sigma e^{(\lambda+\nu) / 2}\left[\left(n-n_{ \pm}\right) \tilde{A}^{(2) l \pm 1 m}(r)+\left(n-n_{ \pm}\right)\left(n-n_{ \pm}-1\right) B^{(2) l \pm 1 m}(r)\right] \\
& \tilde{D}^{(2) l \pm 1 m}\left[Z_{R W}\right]=-\mathrm{i} \sigma \frac{2 e^{(\lambda+\nu) / 2}}{r} c_{s}^{-2}\left[g^{l m \prime}(r)+\left(\nu^{\prime}-\frac{2}{r}+(n+1) \frac{e^{\lambda}}{r}\right) g^{l m}(r)\right] \\
& -\mathrm{i} \sigma \frac{2 e^{(\lambda+\nu) / 2}}{r} c_{s}^{-2}\left[-2\left(n-2 n_{ \pm}-2\right) \chi^{(1) l \pm 1 m}(r)\right. \\
& \left.+\left(n-n_{ \pm}-1\right) \tilde{\alpha}^{(1) l \pm 1 m}(r)-\eta^{(1) l \pm 1 m}(r)\right] \\
& -\mathrm{i} \sigma \frac{e^{(\lambda+\nu) / 2}}{2} c_{s}^{-2}\left[\left(n-n_{ \pm}\right) \tilde{A}^{(2) l \pm 1 m}(r)+\left(n-n_{ \pm}\right)\left(n-n_{ \pm}-1\right) B^{(2) l \pm 1 m}(r)\right] \\
& +\mathrm{i} \sigma \frac{e^{(3 \lambda-\nu) / 2}}{2}\left[\left(n-n_{ \pm}\right) \tilde{A}^{(0) l \pm 1 m}(r)+\left(n-n_{ \pm}\right)\left(n-n_{ \pm}-1\right) B^{(0) l \pm 1 m}(r)\right]
\end{aligned}
$$

where $f, g, \xi^{(J)}, \tilde{\alpha}^{(J)}, \tilde{\beta}^{(J)}, \eta^{(J)}, \zeta^{(J)}, C^{(I)}, \tilde{A}^{(I)}, B^{(I)}$ are quantities which depend on the perturbations $H_{2}^{l m}$, $K^{l m}$, etc., and which are given in Appendix B of K1. 
At the surface of the star we compute $H_{0}^{l m}$ and the other perturbations in terms of $H_{2}^{l m}, K^{l m}, Z_{R W}^{l m}$. We impose the vanishing of the Lagrangian pressure perturbation (see [9]). This reduces the number of freely assigned constants from three (times $L-|m|+1$ ), which correspond to the three independent solutions (B14)-(B16), to two (times $L-|m|+1)$, i.e. $N=2$ as discussed in Section IA2.

Finally, the equations in vacuum are, as in K1,

$$
\begin{aligned}
L_{\text {ext }}^{(J) l m}\left[H_{0}, \tilde{H}_{1}, K\right] & =\frac{\omega}{\sigma}\left(m \hat{E}^{(J) l m}\left[H_{0}, \tilde{H}_{1}, K\right]+\frac{Q_{l-1 m} \hat{D}^{(J) l-1 m}\left[Z_{R W}\right]}{n-n_{-}}+\frac{Q_{l+1 m} \hat{D}^{(J) l+1 m}\left[Z_{R W}\right]}{n-n_{+}}\right) \\
L^{l m}\left[Z_{R W}\right] & =\frac{\omega}{\sigma}\left(m \hat{N}^{l m}\left[Z_{R W}\right]-\frac{Q_{l-1 m} \hat{F}^{l-1 m}\left[H_{0}, K\right]}{n-n_{-}}-\frac{Q_{l+1 m} \hat{F}^{l+1 m}\left[H_{0}, K\right]}{n-n_{+}}\right)
\end{aligned}
$$

$(J=1, \ldots, 4)$ where $L_{\text {ext }}^{(J)}\left[H_{0}, \tilde{H}_{1}, K\right], L^{l m}\left[Z_{R W}\right]$ are the operators defined in (B3)-(B10), (B12), and the expressions of $\hat{E}^{(J) l m}\left[H_{0}, K\right], \hat{D}^{(J) l m}\left[Z_{R W}\right], \hat{N}^{(J) l m}\left[Z_{R W}\right], \hat{F}^{(J) l m}\left[H_{0}, K\right]$ are given in $\mathrm{K} 1$.

When $r \gg R$, the background spacetime is with good approximation spherically symmetric, because the terms due to rotation decrease faster than the "Schwarzschild-like" components (see for instance 30, Chap. 19). Therefore, spacetime perturbations satisfy the Zerilli and the Regge-Wheeler equations.

In this limit, equation (B29) becomes the Regge-Wheeler equation for the function $Z_{R W}^{l m}$, whereas the Zerilli function $Z_{Z e r}^{l m}$ is related to the solution of equation (B28) by (B11). At radial infinity, the amplitude of the stationary wave $\left(A_{Z \text { er in }}^{l m}(\sigma), A_{R W \text { in }}^{l m}(\sigma)\right)$ can be computed in terms of $Z_{Z \text { er }}^{l m}$ and $Z_{R W}^{l m}$. We can then apply the stationary wave approach described in Section $\Pi$ A and in Appendix A 3.

Equations (B23), B28), B29) can be integrated using the spectral decomposition in Chebyshev's polynomials as explained in Section IIB. There is a main difference with respect to the example described in Appendix A3, which refers to the axial equation for a non rotating star. While the matrix A33 is block-diagonal - each block corresponding to a value of $l$ - the matrix representing equations (B23), (B28), (B29) presents, in addition to the block-diagonal terms, components of order $O(\epsilon)$, which couple $l \leftrightarrow l \pm 1$.

All equations in this paper have been checked using Maple, and we have made several cross checks in order to be sure that the Fortran implementation of the long expressions (B24)-(B27) are correct.

[1] S. Chandrasekhar, Phys. Rev. Lett. 24, 611 (1970); J.L. Friedman, B.F. Schutz, Astroph. J. 222, 281 (1978).

[2] K.S.Thorne, A.Campolattaro Astroph. J. 149, 591 (1967).

R.Price, K.S.Thorne, Astroph. J. 155, 163 (1969).

K.S.Thorne Phys. Rev. Lett. 21, 320 (1968).

A.Campolattaro, K.S.Thorne, Astroph. J. 159, 847 (1973).

J.R.Ipser, K.S.Thorne, Astroph. J. 181, 181 (1973).

[3] S.Chandrasekhar, V.Ferrari, Proc. R. Soc. Lond. A432, 247 (1991).

[4] S.Chandrasekhar, V.Ferrari, Proc. R. Soc. Lond. A434, 449 (1991). S.Chandrasekhar, V.Ferrari, Proc. R. Soc. Lond. A437, 133 (1992).

[5] L. Lindblom, S. Detweiler, Astroph. J. Suppl. 53, 73 (1983).

N. Andersson, K.D. Kokkotas, Phys. Rev. Lett. 77, 4134 (1996).

N. Andersson and K. D. Kokkotas, Mon. Not. R. Astron. Soc. 320, 307 (1999).

O. Benhar, V. Ferrari, L. Gualtieri, Phys. Rev. D 70, 124015 (2004).

C.W. Yip, M.-C.Chu, P.T. Leung, Astroph. J. 513, 849 (1999).

Y. Kojima and K. Sakata, Prog. Teor. Phys. 108, 801 (2002).

H. Sotani and T. Harada, Phys. Rev. D 68, 024019 (2003).

H. Sotani, K. Khori and T. Harada, Phys. Rev. D 69, 084008 (2004).

O. Benhar, V. Ferrari, L. Gualtieri, S. Marassi, to appear on Gen. Rel. Grav.

[6] H. Sotani and T. Harada, Phys. Rev. D 68, (2003) 024019;

[7] Y. Kojima, Phys. Rev. D 46, 4289 (1992).

[8] Y. Kojima, Astrophys. J. 414, 247 (1993).

[9] Y. Kojima, Prog. Theor. Phys. Suppl. 128, 251 (1997).

[10] J. Ruoff, A. Stavridis, K.D. Kokkotas, Mon. Not. R. Astron. Soc. 330, 1027 (2002).

[11] L. Villain, S. Bonazzola, Phys. Rev. D 66, 123001 (2002).

[12] V. Ferrari, L. Gualtieri, J.A. Pons, A. Stavridis, Mon. Not. R. Astron. Soc. 350, 763 (2004).

[13] L. Villain, S. Bonazzola, P. Haensel, Phys. Rev. D71, 083001 (2005).

[14] J.A. Pons, L. Gualtieri, J.A. Miralles, V. Ferrari, Mon. Not. R. Astron. Soc. 363, 121 (2005). 
[15] A. Stavridis, A. Passamonti, K. Kokkotas, Phys. Rev. D 75, 064019 (2007);

A. Passamonti, A. Stavridis, K. Kokkotas, arXiv:0706.0991 [gr-qc].

[16] K.H. Lockitch, J.L. Friedman, N. Andersson, Phys.Rev. D 68, 124010 (2003).

[17] S. Yoshida, Y. Eriguchi, Astrophys. J. 515, 414 (1999);

S. Yoshida, Y. Eriguchi, Mon. Not. R. Astron. Soc. 322, 389 (2001);

S. Yoshida, L. Rezzolla, Astrophys. J. 568, L41 (2002);

S. Yoshida, S. Yoshida, Y. Eriguchi, Mon. Not. R. Astron. Soc. 356, 217 (2005);

N. Stergioulas, T.A. Apostolatos, J.A. Font, Mon. Not. R. Astron. Soc. 352, 1089 (2004).

[18] J.A. Font et al., Phys. Rev. D 65, 084024 (2002).

[19] N. Stergioulas, J.L. Friedman, Astrophys. J. 492, 301 (1998).

[20] S.M. Morsink, N. Stergioulas, S.R. Blatting, Astrophys.J. 510, 854 (1999).

[21] H. Dimmelmeier, N. Stergioulas, J.A. Font, Mon. Not. R. Astron. Soc., 368, 1609 (2006).

[22] J.A. Font, H. Dimmelmeier, A. Gupta, N.Stergioulas, Mon. Not. R. Astron. Soc. 325, 1463 (2001).

[23] K.S. Thorne, Astroph. J. 158, 1 (1969).

[24] S. Chandrashekhar, V. Ferrari and R. Winston, Proc. R. Soc. Lond. A434, 635 (1991);

V. Ferrari Phil. Trans. R. Soc. Lond A340, 423 (1992).

[25] J.M. Bardeen, Astrophys. J. 162, 71 (1970).

[26] J.B. Hartle, D.H. Sharp, Astrophys. J. 147, 317 (1967).

[27] J.B. Hartle, Astrophys. J. 150, 1005 (1967).

[28] T. Regge, J.A. Wheeler, Phys. Rev. 108, 1063 (1957).

[29] F.J. Zerilli, Phys. Rev. D 2, 2141 (1970).

[30] C.W. Misner, K.S. Thorne, J.A. Wheeler, Gravitation, W.H. Freemand and Company, New York (1973).

[31] S. Bonazzola, E. Gourgoulhon and J.A. Marck, J. Comput. Appl. Math., 109, 433 (1999). P. Grandclement and J. Novak, arXiv:0706.2286 [gr-qc].

[32] W.H.Press, S.A.Teukolsky, W.T.Vetterling, B.P.Flannary, Numerical Recipes, Cambridge University Press (1992).

[33] S. Bonazzola, E. Gourgoulhon, J.A. Marck, Phys. Rev. D 58, 104020 (1998).

[34] L. Samuelsson, N. Andersson and A. Maniopoulos, Class. Quant. Grav. 24, 4147 (2007). 